Bulletin of Mathematical Biology (2002) 64, 501-530

doi:10.1006/bulm.2002.0283

Available online at http://www.idealibrary.com on IDE $\mathbf{L}^{\mathbb{1}}$

\title{
A Numerical Approach to the Study of Spatial Pattern Formation in the Ligaments of Arcoid Bivalves
}

\author{
ANOTIDA MADZVAMUSE \\ Oxford University Computing Laboratory, \\ Wolfson Building, Parks Road, \\ Oxford OX1 3QD, \\ U.K.
}

ROGER D. K. THOMAS

Department of Geosciences,

Franklin \& Marshall College,

Lancaster, Pennsylvania,

17604-3003,

U.S.A.

\section{PHILIP K. MAINI}

Centre for Mathematical Biology, Mathematical Institute,

University of Oxford,

24-29 St Giles',

Oxford OX1 3LB,

U.K.

\author{
ANDREW J. WATHEN \\ Oxford University Computing Laboratory, \\ Wolfson Building, Parks Road, \\ Oxford OX1 3QD, \\ U.K.
}

In this paper, we employ the novel application of a reaction-diffusion model on a growing domain to examine growth patterns of the ligaments of arcoid bivalves (marine molluscs) using realistic growth functions. Solving the equations via a novel use of the finite element method on a moving mesh, we show how a reactiondiffusion model can mimic a number of different ligament growth patterns with modest changes in the parameters. Our results imply the existence of a common mode of ligament pattern formation throughout the Arcoida. Consequently, arcoids that share a particular pattern cannot be assumed, on this basis alone, to share an immediate common ancestry. Strikingly different patterns within the set can easily be generated by the same developmental program. We further show how the model can be used to make quantitatively testable predictions with biological implications.

(c) 2002 Society for Mathematical Biology. Published by Elsevier Science Ltd. All rights reserved. 


\section{INTRODUCTION AND BIOLOGICAL BACKGROUND}

Colour patterns on the exterior surfaces of molluscan shells are formed by pigment secreted in a narrow zone at the growing margin of the shell. Consequently, these patterns develop incrementally, as records of activity in the growth zone accumulate in the course of time. The basic elements of these patterns are colour bands running perpendicular, parallel, or oblique to the direction of domain growth. These patterns can be modelled by systems of coupled reaction-diffusion equations involving two, and sometimes three or more, morphogens (Meinhardt and Klinger, 1987; Meinhardt, 1995).

Analogous patterns that we investigate in this paper are generated in the course of growth of the ligaments of arcoid bivalves (Fig. 1). These growth patterns are comparable with shell colour patterns in that arcoid ligaments typically consist of alternating sheets or strips of two different kinds of material, secreted in this case along the dorsal margin of the growing shell. Thus, it seems highly probable that similar molecular mechanisms, involving alternating activation and inhibition, are involved in the development of arcoid ligaments and shell colour patterns. However, the two sets of patterns differ in relation to the boundaries of the fields where they are generated. The elements of shell colour patterns are generally small in scale relative to the surfaces on which they occur, so edge effects are limited, apart from those due to growth at the expanding shell margin. In contrast, the growth pattern of the arcoid ligament is constrained by the size and shape of its area of attachment to the shell. Consequently, the boundaries of this domain directly affect the scale and orientation of ligament growth patterns.

Shells of the class Bivalvia typically consist of two calcareous valves, connected by an elastic ligament. The order Arcoida is a major subgroup of this class, characterised by shells with numerous, small hinge teeth and ligaments forming chevrons or other structurally related patterns of attachment to the valves, as well as various aspects of shell microstructure and their soft tissues. Growth patterns of the ligament play an important part in the recognition of evolutionary relationships among members of this group. The family Arcidae includes bivalves with elongated shells, long straight hinges, and a ligament composed of chevrons that are sometimes discontinuous, extending along the entire length of the hinge. Members of the Glycymerididae are shallow burrowers, with more or less circular shells, hinge teeth forming an arc around the dorsal margin, and a fully developed chevron ligament. The Limopsidae consists of small animals, also burrowers, with an arcuate hinge. In this group, the ligament is much reduced, confined to a triangular pit immediately below the umbones - the earliest-formed 'beaks' of the valves-away from which the shell rotates as a result of its spiral growth. The Noetiidae is a family of rather varied forms, nestlers or shallow burrowers, with ligaments that are most divergent from the general pattern. In all cases, the ligament consists of vertical strips, but these may extend along the length of the hinge, as in Noetia, along part of the hinge in Striarca (Fig. 2) or it may be confined to a small pit, as in Arcopsis. 


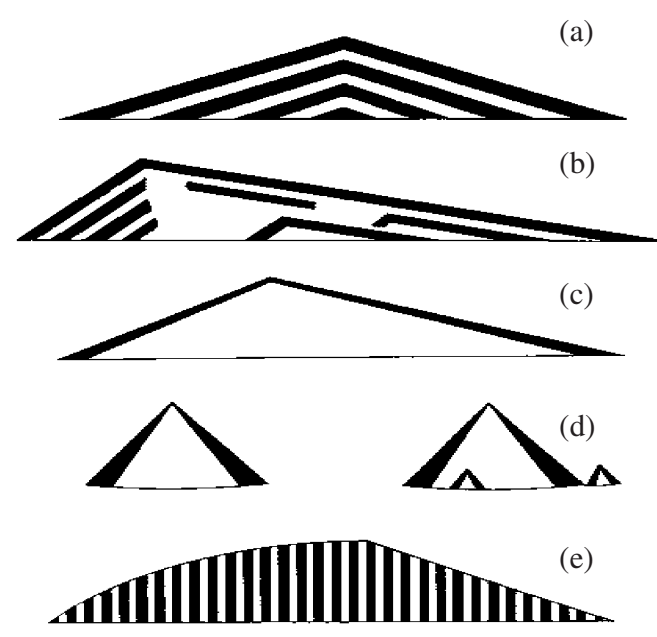

Figure 1. Representative growth patterns of arcoid ligaments. (a) Glycymeris; (b) Arca; (c) Cucullaea; (d) Limopsis; (e) Limopsis variant, not uncommon in some species; and (f) Noetia. These patterns are the subject of our study.
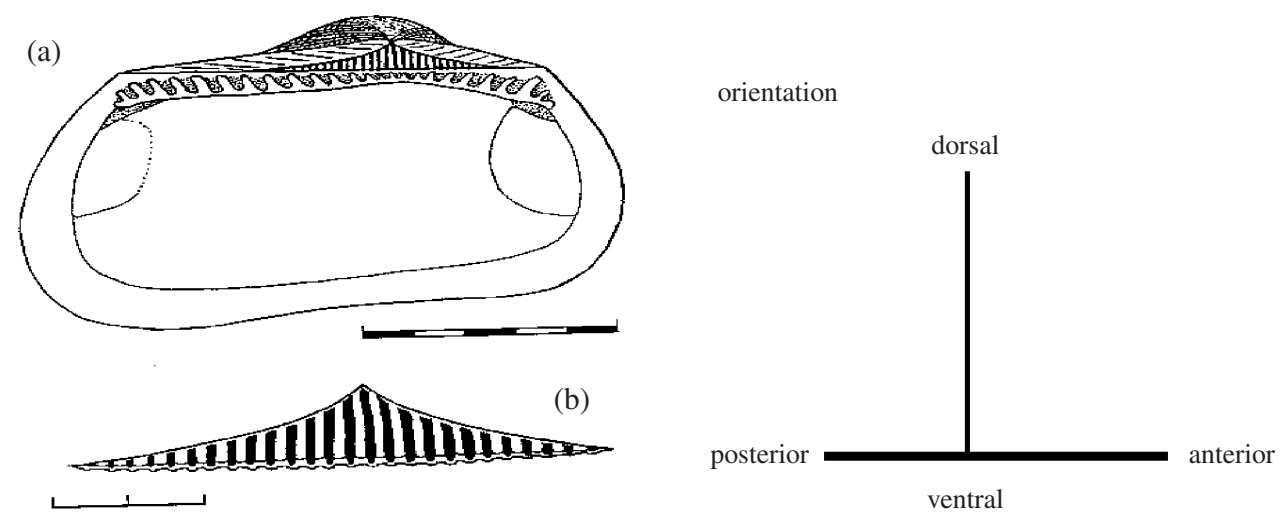

Figure 2. (a) Left valve of Striarca lactea showing allometric growth of the ligament. This growth has been achieved by expansion from a small triangular area to occupy a large proportion of the cardinal area in the adult shell. Scale bar, $5 \mathrm{~mm}$. (b) Enlargement showing vertical strips of lamellar ligament embedded in fibrous ligament and not directly attached to the shell. Scale bar, $2 \mathrm{~mm}$. 


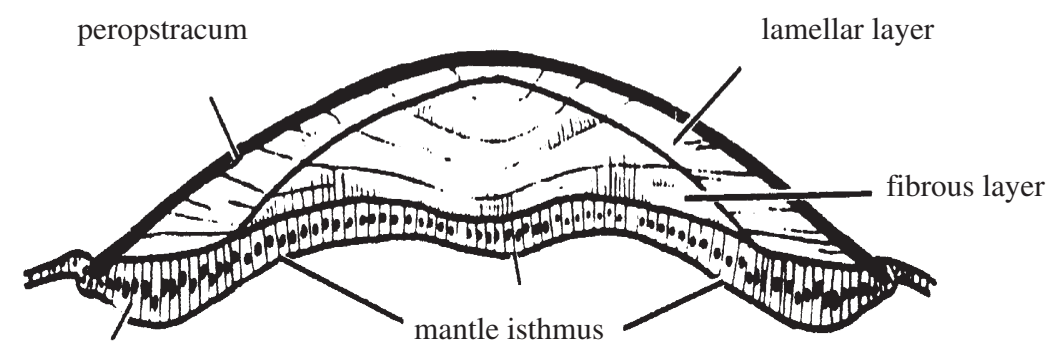

outer mantle fold

Figure 3. Bivalve ligament in longitudinal section. Adapted from Fig. 51, Trueman (1969).

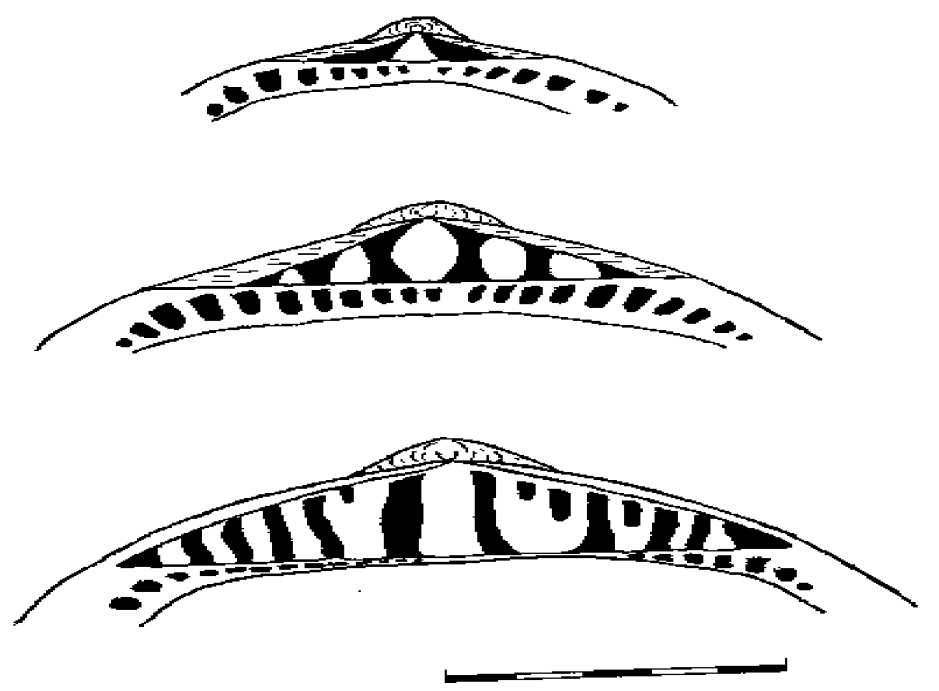

Figure 4. Ligaments of Limopsis marionensis from the South Atlantic, near the Falkland Islands. Scale bar, $1 \mathrm{~cm}$.

There are other families, some represented only in the fossil record, but these are the ones with which we are concerned here. Conventionally, we will refer to the Arcoida or to the Limopsidae, implying the order or the family as a whole, and we will refer informally to members of these higher taxa as arcoids or limopsids.

The bivalve ligament is the uncalcified part of the shell by which the two valves are attached, dorsally. It is secreted at the mantle isthmus (Fig. 3), where the sheets of mantle tissue responsible for secreting the two valves meet. Bivalve shells grow by expanding the shell around the margin and by the addition of new layers of material to their interior surfaces.

The ligament, consisting largely of tanned proteins, grows across the dorsal junction between the two valves. It increases in height by addition of material to its base and in length by extension, posteriorly (opisthodetic), anteriorly (prosodetic), 
or both (amphidetic), along the mantle isthmus. The ligament consists of lamellar and fibrous elastic materials, which serve to open the valves. The antagonistic relationship between the ligament and adductor muscles ventral to the hinge axis enables the animal to open and close its shell. When the shell is closed, lamellar material dorsal to the hinge axis is stretched and fibrous material below it is compressed. When the adductor muscles relax, strain in both parts of the ligament is released and the shell opens (Trueman, 1969; Thomas, 1976).

The purpose of this paper is to enhance our understanding of the process that controls pattern formation in the development of arcoid ligaments, by modelling the growth patterns described in Section 2. Section 3 focuses on the relationship between our models and the actual growth of the organisms. We aim to demonstrate how a fairly simple model can generate the observed variety of ligament growth patterns, under the control of relatively few conditions and parameters. In particular, we show that the role of domain growth, coupled with appropriate boundary conditions, is crucial in the formation of robust growth patterns. In Sections 4 and 5 , we present the model equations used in our investigations and the numerical technique we applied to solve these equations on a moving grid. We present and discuss our numerical results in Sections 6 and 7. Predictions and experimental tests are presented in Section 8. Finally, we summarize the implications of our study in Section 9.

\section{Observations of Growth Patterns of the Arcoid Ligaments}

The ligaments of arcoid bivalves typically consist of oblique sheets of lamellar and fibrous material. These alternate along the hinge, so their attachments form characteristic chevron patterns on each valve, between the hinge plate and the umbones. New elements are added to this pattern at or near the middle of the growth zone, as the ligament expands anteriorly and posteriorly, along the hinge axis (Newell, 1937). The lamellar material is elastic in its response to extensional and compressional forces. The fibrous material responds elastically only to compressional forces. Away from the region of ligament growth, the brittle fibrous ligament splits above the hinge axis, as the growing valves diverge. The lamellar ligament is stretched between the valves, producing the tension that causes them to spring open when the muscles relax. Further dorsally, the lamellar ligament is stretched beyond its elastic limit and it tears. The addition of new elements to this pattern ensures that some new, unbroken sheets of lamellar material are always present. Together with strong, positive allometric growth of most arcoid ligaments, this ensures that the ligament remains strong enough, in spite of its dorsal breakage, to open the shell as it gets larger (Thomas, 1976).

In Glycymeris and Arca, oblique sheets of lamellar and fibrous material diverge anteriorly and posteriorly, away from a point below the umbones [Fig. 1(a) and 1(b)]. As the ligament expands ventrally, secretion at the mid-point of the pattern 


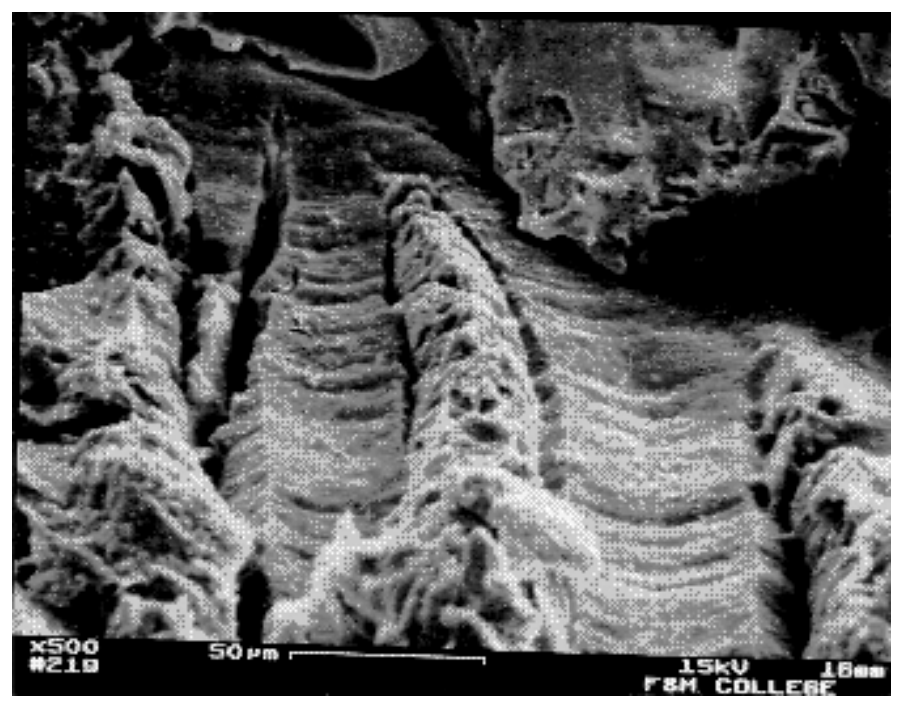

Figure 5. Scanned electron micrograph of the ligament of Striarca lactea. Observe that strips of lamellar ligament are inserted in grooves in the fibrous ligament, subsequently turning away from the margin of their attachment area to assume a vertical orientation.

switches periodically between the two types of material. This process is regulated in such a way as to maintain uniform widths and spacing of the sheets of lamellar and fibrous material [Fig. 1(a)]. In Limopsis, the pattern is normally reduced to a single pair of anterior and posterior lamellar sheets, separated by an expanding wedge of fibrous ligament [Fig. 1(d)]. This ligament develops by prolongation of the earliest stages of the pattern observed in Glycymeris. Deviations from these regular patterns occur to a greater extent in some taxa [Fig. 1(b) and 1(e)] than in others.

The growth pattern of the noetiid ligament [Figs 1(f) and 2(b)] departs from those of all other arcoid families in that here the lamellar material is inserted in vertical strips, as opposed to forming oblique sheets. This is accomplished by keeping the site of secretion of each lamellar strip fixed, instead of migrating along the hinge axis during the course of shell growth. Consequently, new elements are added to the noetiid pattern at its anterior and posterior extremities, rather than at a point of divergence beneath the umbones as in other arcoids.

The ligaments of an unusual series of specimens of Limopsis marionensis show how the noetiid pattern may have arisen. As juveniles, members of this population have typical limopsid ligaments. However, the adult shells, which are strikingly large for this genus, develop multiple, vertical strips of lamellar ligament [Fig. 4; Thomas et al. (2000)]. New strips of lamellar material are added at the anterior and posterior extremities of the growing ligament, as in the Noetiidae. The close comparison between the growth of the ligament in this aberrant population of L. marionensis and that of the noetiids is confirmed by the ligament of Striarca lactea. In Fig. 5, strips of lamellar material can be seen to grow obliquely when they are first 

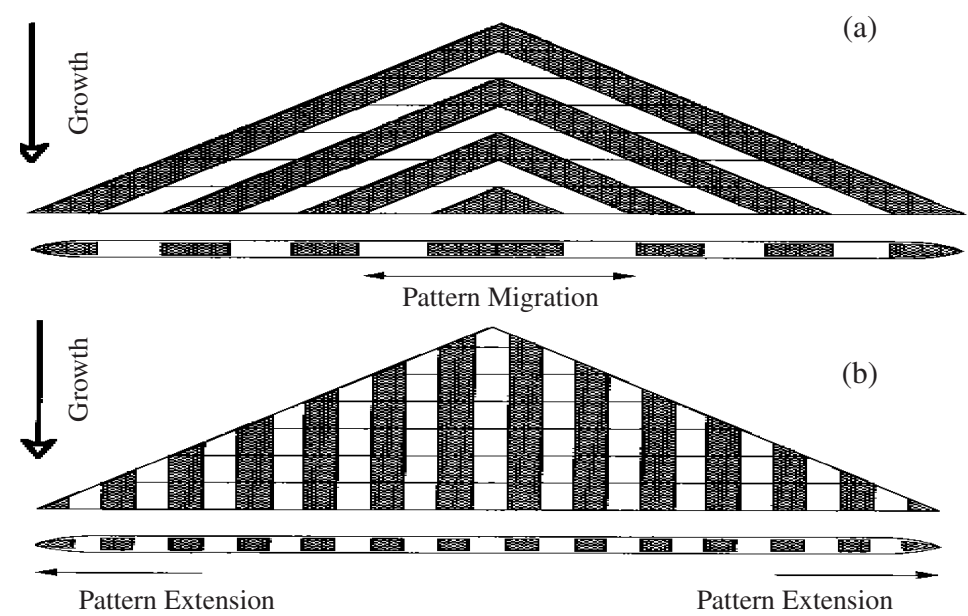

Figure 6. Growth patterns of (a) the duplivincular ligament typical of Glycymeris; and (b) the noetiid ligament. Each diagram shows a cross-sectional view of the ligament (dark shading) as it is inserted on the attachment area of each valve and a dorsal view of the mantle isthmus, where new ligamental material is secreted. This illustrates clearly the one dimensionality of the problem.

introduced at the margins of the ligament. As the ligament continues to expand, these strips turn towards a vertical orientation, which they achieve when they reach the width characteristic of the pattern observed in the adult ligament.

\section{Modelling of the Growth Patterns: Assumptions}

Observations of the shells of living and extinct arcoids indicate that, while the patterns of lamellar and fibrous material vary, growth of the ligament as a whole is always directed ventrally, away from the umbones. Typical growth patterns of a glycymerid and a noetiid are illustrated schematically in Fig. 6, which shows how the chevrons and vertical strips are formed. In each case, a horizontal cross-section or ventral view of the base of the ligament is shown, indicating the pattern of secretion of lamellar and fibrous material at any stage in the growth of the ligament.

We have no direct observations of growth rates of Glycymeris, Limopsis, or other arcoids. Bivalves in general live for at least a year; larger species commonly live for 10-15 years, or much longer in some cases. Annual growth rings, reflecting seasonal changes in growth rate, can be recognized in many species. However, spawning and disturbance by storms can give rise to similar rings, so these do not always provide a reliable measure of the age of the animal. Daily growth increments can be recognised in the shell microstructure of many shallow-water species. Traces of these increments can often be seen as horizontal lines on the surface of the ligamental attachment area, recording its ventrally directed growth and that of the ligament itself. 
Typically, the growth patterns of arcoid ligaments display uniform spacing of the oblique or vertical sheets of lamellar material (Fig. 6). It is important to note that although these alternations are uniformly spaced, the rate at which they are formed differs from the rate at which the shell grows. Shell growth occurs along a path that closely approximates a logarithmic spiral (Thompson, 1917), which is expressed in the curvature of the ligamental attachment surface (Thomas, 1976). This implies the existence of two different growth processes. There is a function which determines the height of the ligamental attachment area, or any other measure of its overall size. At the same time, there is another function that controls pattern formation within the ligament. The domain as a whole grows rapidly at first and more slowly at later stages (see Section 6). However, new elements of the glycymerid growth pattern appear at regularly spaced points along a straight line extending from the centre of the spiral (the umbo) to its latest growth increment, at the base of the ligament. Juvenile noetiids exhibit ligament growth patterns that are very similar to those of Limopsis, with a single pair of anterior and posterior layers. In the case of $L$. marionensis noted above, varying numbers of irregular vertical strips of lamellar ligament develop, as these become adults. This clearly shows that the patterns are not predetermined at an early developmental stage and fixed for life. Rather, the patterns change continuously as the ligament grows. This indicates that pattern formation in the arcoid ligament is closely linked to its growth as a whole, implying that the pattern is under the control of a growing domain.

Sheets of lamellar and fibrous ligament are introduced medially by insertion or they are added at the anterior and posterior extremities of the ligament, when sufficient space is created. This phenomenon of insertion and addition to patterns has been observed in one-dimensional (1D) reaction-diffusion models (Turing, 1952) by a number of authors (Kondo and Asai, 1995; Meinhardt, 1995, Fig. 2.6; Painter, 1997; Crampin, 2000). Kiskaddon (1996) considered a simple 1D harmonic bifurcation model which treats the mantle isthmus as a finite, expanding space that can accommodate an integral number of waves. His approach employs morphogenetic rules like those used by Oster et al. (1988) to model patterns of cartilage condensation in the growth of vertebrate limbs. Kiskaddon's model is based on a sine function. This is assumed to represent the net effect of activating and inhibiting processes that are involved in generating the repetition of sheets to form a typical chevron ligament. A threshold value of the sine function acts as a switch, controlling changes in the type of secretion. Consequently, it determines the relative widths of the lamellar sheets and the fibrous material that is laid down between them. Simulations produced by this model confirm that one or two simple changes in the instructions suffice to derive the noetiid ligamental growth pattern from a developmental system that was originally programmed to produce the chevron patterns of other arcoids (Thomas et al., 2000). However, this model neither specifies the variables that underlie the sine function, nor does it show how the function persists and changes to produce biologically realistic growth patterns. Here, we investigate a reaction-diffusion model that more faithfully represents plausible 
mechanisms and we show that it can explain how the various growth patterns of arcoid ligaments may have arisen.

Other mechanisms, apart from reaction-diffusion kinetics, may control the development of patterns of this sort. Murray (1993) has reviewed models based on mechanochemical cues. Ermentrout et al. (1986) modeled long-range patterns of neural activation and inhibition as potential controls of colour pattern formation in molluscan shells. Mathematically, these models are all analogous, employing systems of equations that reflect the general underlying patterning principle of short-range activation, long-range inhibition (Geirer and Meinhardt, 1972). Consequently, the validity of our models and the inferences we draw from them are largely independent of the specific mechanisms that are involved in the development of arcoid ligaments. These mechanisms have not so far been identified.

\section{Model Equations}

For illustrative purposes we restrict ourselves here to the application of the simple partial differential equation (PDE) Schnakenberg (1979) reaction model given, in non-dimensional form, by

$$
\begin{aligned}
& u_{t}=\gamma\left(a-u+u^{2} v\right)+\nabla^{2} u, \\
& v_{t}=\gamma\left(b-u^{2} v\right)+d \nabla^{2} v,
\end{aligned}
$$

where $u(x, t)$ and $v(x, t)$ are chemical concentrations associated with the secrection of lamellar and fibrous ligament, respectively, at position $x$ and time $t ; d$ is the ratio of diffusion coefficients of $D_{1}$ and $D_{2}$ (typically of the order of between $10^{-8}$ $10^{-10} \mathrm{~cm}^{2} \mathrm{~s}^{-1}$ ); and $a$ and $b$ are reaction kinetic parameters. Note that this model is a special case of that given by equations (11a), (11b) of Geirer and Meinhardt (1972). The scaling parameters are defined as

$$
T=\frac{L_{x}^{2}}{D_{1}}, \quad \gamma=\frac{k_{2} L_{x}^{2}}{D_{1}} \quad \text { and } \quad d=\frac{D_{2}}{D_{1}} .
$$

Here $L_{x}$ is the length scale and $k_{2}$ a reaction kinetic rate. In our case of arcoid ligaments, $L_{x}$ is of order $\mathrm{mm}$.

In all our simulations, typically, boundary conditions are either Neumann for both $u$ and $v$ or Neumann for one component and Dirichlet for the other or Dirichlet for both components. functions but in practice these are defined as small random perturbations about the uniform homogeneous steady state, if it exists.

\section{The Finite Element Method on a Moving Mesh}

The generality of the moving finite element has the advantage over most numerical methods in that dynamically deforming complex geometries are dealt with 
easily and efficiently without much change in the numerical code. Although this sophistication is not required here, we have been developing a numerical technique that can be applied to a wide variety of problems with arbitrarily continuously deforming domains. There is essentially no loss of efficiency in using these methods over simpler and less widely applicable ones.

The basic idea behind the application of the finite element method to our problem is to discretise the given continuous problem (4.1) and (4.2) with infinitely many degrees of freedom to obtain a discrete problem or system of equations with only finitely many unknowns. The discretisation process starts from the reformulation of the given PDE to an equivalent weak form over a space $V$. Multiplying (4.1) and (4.2) by $w \in V$ and applying Green's theorem we have the following problem: find $u, v \in V$ such that

$$
\begin{aligned}
& \left(u_{t}, w\right)=\left(\gamma\left(a-u+u^{2} v\right), w\right)+\left(\nabla^{2} u, w\right), \\
& \left(v_{t}, w\right)=\left(\gamma\left(b-u^{2} v\right), w\right)+d\left(\nabla^{2} v, w\right),
\end{aligned}
$$

for all $w \in V$ where

$$
(u, w)=\int_{\Omega(t)} u w d \Omega(t)
$$

is the $L_{2}$-inner product.

To obtain a problem that can be solved numerically $V$ is replaced by $V^{h}$, a finitedimensional subspace consisting of simple functions depending only on finitely many parameters. We use the well-known Galerkin method (in which both the approximate solution $u^{h}$ and the test function $w^{h}$ lie in the finite-dimensional approximation space $V^{h}$ ). Following standard use of the divergence theorem, this leads to the following finite-dimensional problem: find $u^{h}, v^{h} \in V^{h}$ such that

$$
\begin{aligned}
& \left(u_{t}^{h}, w^{h}\right)=\left(\gamma\left(a-u^{h}+u^{h^{2}} v^{h}\right), w^{h}\right)-\left(\nabla u^{h}, \nabla w^{h}\right), \\
& \left(v_{t}^{h}, w^{h}\right)=\left(\gamma\left(b-u^{h^{2}} v^{h}\right), w^{h}\right)-d\left(\nabla v^{h}, \nabla w^{h}\right),
\end{aligned}
$$

for all $w^{h} \in V^{h}$ where Neumann boundary conditions have been applied for illustrative purposes ${ }^{\dagger}$. This semi-discrete problem is a nonlinear system of equations. The finite element method in its simplest form is a Galerkin method characterized by the following basic aspects in the construction of the space $V^{h}$ :

(i) define a triangulation $\mathcal{T}^{h}$ over the solution domain given by a finite union of finite elements $K \in \mathcal{T}^{h}$,

(ii) $V^{h}$ is the linear span of piecewise polynomials for which there exists a basis function having local support.

\footnotetext{
${ }^{\dagger}$ More complicated boundary conditions can also be applied (Saad, 1996).
} 
Let $u^{h}$ and $v^{h}$ be the finite element approximation to $u$ and $v$ respectively, defined as

$$
u^{h}(\mathbf{x}, t)=\sum_{i=0}^{N+1} u_{i}^{h}(t) \alpha_{i}(\mathbf{x}, \mathbf{a}(t)) \quad \text { and } \quad v^{h}(\mathbf{x}, t)=\sum_{i=0}^{N+1} v_{i}^{h}(t) \alpha_{i}(\mathbf{x}, \mathbf{a}(t))
$$

where $\mathbf{x} \in \mathbb{R}^{m}$ indicates the spatial coordinates and $\mathbf{a}(t)$ represents the grid in time, that is the vector of nodal positions at time $t$. This grid is given by $\mathbf{a}(t)=$ $\left(a_{0}(t), a_{1}(t), \ldots, a_{N+1}(t)\right)^{T}$. Here, $\alpha_{i}(\mathbf{x}, \mathbf{a}(t))$ is the piecewise linear hat function, which is defined to be the unique linear spline on the grid such that $\alpha_{i}\left(\mathbf{a}_{j}, \mathbf{a}(t)\right)=$ $\delta_{i, j}$. Linear spline functions are chosen in preference to cubic splines simply because they are sufficient in our case and more easy to compute with. We first calculate the time derivative of the finite element approximations $u^{h}$ and $v^{h}$ respectively. For example, the time derivative of $u^{h}$ is given by

$$
\frac{\partial u^{h}}{\partial t}=\sum_{i=0}^{N+1} \frac{\partial}{\partial t}\left(u_{i}^{h}(t) \alpha_{i}(\mathbf{x}, \mathbf{a}(t))\right) .
$$

Applying the product and the chain rule respectively, we obtain (Jimack and Wathen, 1991)

$$
\frac{\partial u^{h}}{\partial t}=\sum_{i=0}^{N+1} \frac{d u_{i}^{h}}{d t} \alpha_{i}+\sum_{i=0}^{N+1} \beta_{i}^{T} \dot{\mathbf{a}}_{i}(t)
$$

where ${ }^{*}=\frac{d}{d t}$. It can be observed that

$$
\beta_{i}=\sum_{j=0}^{N+1} u_{j}^{h} \nabla_{\mathbf{a}_{j}} \alpha_{i}=\nabla_{\mathbf{a}_{j}} \sum_{j=0}^{N+1} u_{j}^{h} \alpha_{i}=\nabla_{\mathbf{a}_{j}} u^{h}=\frac{\partial u^{h}}{\partial \mathbf{a}_{i}} .
$$

Here

$$
\nabla_{\mathbf{a}_{j}}=\frac{\partial}{\partial \mathbf{a}_{i}}=\left(\frac{\partial}{\partial a_{i, 0}}, \frac{\partial}{\partial a_{i, 1}}, \cdots, \frac{\partial}{\partial a_{i, m}}\right)^{T}
$$

where $i$ indicates the nodal position and $m$ is the dimension. Baines and Wathen (1988) proved that

$$
\beta_{i}=\frac{\partial u^{h}}{\partial a_{i}}=\alpha_{i} \frac{\partial u^{h}}{\partial \mathbf{x}}
$$

in multi-dimensions. In particular (5.11) reduces to $\beta_{i}=-\alpha_{i} u_{x}^{h}$ with $\dot{\mathbf{a}}_{i}=\dot{x}_{i}$ in one dimension and $\dot{\mathbf{a}}_{i}=\left(\dot{x}_{i}, \dot{y}_{i}\right)^{T}$ and $\frac{\partial u^{h}}{\partial \mathbf{x}}=\left(u_{x}^{h}, u_{y}^{h}\right)$ in two dimensions. Therefore the time derivative of $u^{h}$ is given by

$$
\frac{\partial u^{h}}{\partial t}=\sum_{i=0}^{N+1}\left[\dot{u}_{i}^{h}-\dot{x}_{i} u_{x}^{h}\right] \alpha_{i}(\mathbf{x}, \mathbf{a}(t))
$$


in one dimension and

$$
\frac{\partial u^{h}}{\partial t}=\sum_{i=0}^{N+1}\left[\dot{u}_{i}^{h}-\left(\dot{x}_{i} u_{x}^{h}+\dot{y}_{i} u_{y}^{h}\right)\right] \alpha_{i}(\mathbf{x}, \mathbf{a}(t))
$$

in two dimensions.

The effect of the growing domain is to add extra terms to the finite element formulation as illustrated in the general case (5.8) and in particular for (5.12) and (5.13) in the one- and two-dimensional (2D) cases respectively. Equations (5.4) and (5.5) together with (5.12) and (5.13) give rise to a semi-discrete system of nonlinear ordinary differential equations. The discretisation of the time derivative and the linearisation of the nonlinear terms is carried out simultaneously. First, the nonlinear terms are linearised by applying the Picard iteration method (Reddy, 1984). We will assume that domain growth occurs slowly (Section 6). This assumption implies that on a moving grid mesh, the term $u^{h^{2}}$ for example, can be written as a product of $u^{h^{(m)}} u^{h^{(m+1)}}$ where $u^{h^{(m)}}$ is the known solution calculated from the previous grid at time $m \Delta t$. To calculate the successive solution $u^{h^{(m+1)}}$, we use the previous solution evaluated on the new grid. The same applies for $v^{h}$ in equation (5.4). $v^{h^{(m)}}$ is evaluated on the new grid. In equation (5.5), $v^{h}$ decouples automatically as it is linear in itself. This form of decoupling together with the Picard iteration method gives rise to symmetric matrices [for specific details refer to Madzvamuse (2000)]. To discretize the linearized ordinary differential equations, we apply the backward Euler finite difference scheme. Although the method is a first order scheme it is unconditionally stable. In one dimension the discretization gives rise to symmetric, tridiagonal and diagonally dominant systems which can be solved using the Thomas algorithm (Morton and Mayers, 1994). In two dimensions we use a preconditioned conjugate gradient method (Saad, 1996). The key effect of the Picard linearization used in this way with backward Euler timestepping is that part of the nonlinear terms is lagged leaving only a linear term at the forward time level hence making the procedure much easier. We have not found the linear convergence of the Picard iteration to be a significant handicap in that very few iterations are needed, typically one Picard iteration with reasonably small time-steps is sufficient.

To model growth, we impose a growth rate function, $r(t)$ on the domain. We know qualitatively that this function must exhibit fast growth at the initial stages and slow growth at later stages. In the absence of precise quantitative data, we choose for illustrative purposes

$$
r(t)=1+\sigma \sqrt{t}
$$

where $\sigma$ is a constant (taken to be 0.02 in our simulations). We define the nodal movement by

$$
x=X(0) r(t)
$$


(a)

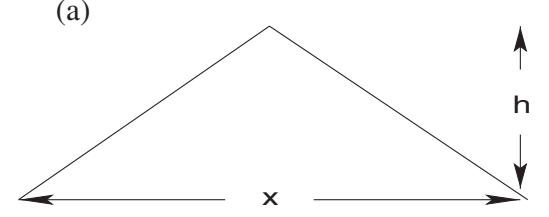

(c)

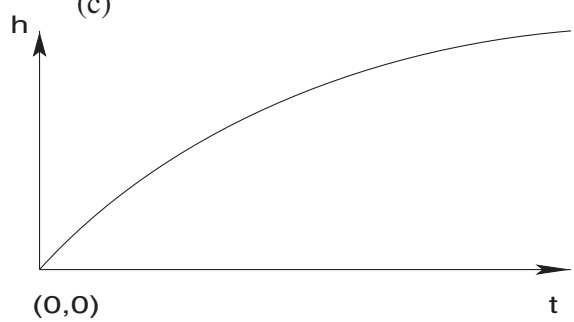

(b)

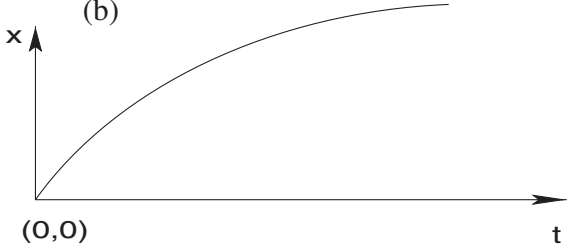

(d)

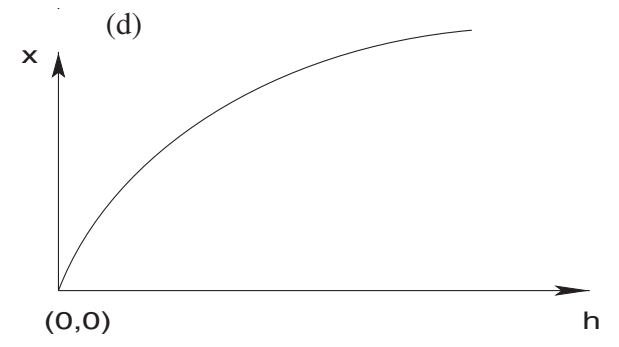

Figure 7. (a) The height of ligamental attachment area as a function of $x$. (b) The growth function of the bivalve: $x=X(0) r(t)$. (c) The growth function of the height in time: $h=\sigma t^{\frac{2}{3}}$ and (d) the growth function of $x$ in terms of the height: $x=X(0)\left(1+\sigma^{\frac{1}{4}} h^{\frac{3}{4}}\right)$.

with flow velocity

$$
\dot{x}=X(0) \dot{r}(t), \quad t>0,
$$

and assume that $X(0)=\{x \in \mathbb{R}:-1 \leq x \leq 1\}=I(0)$ is the initial grid at time $t=0$.

Let $h(t)$ be the function describing the height of the ligamental attachment area, i.e. representing the rate at which lamellar layers are created [Fig. 7(a)]. We define, for simplicity, $h(t)=\sigma_{1} t^{k}(0<k<1)$ a polynomial function in time which does not blow-up in finite time. In order for this to be consistent with our assumptions in Section 3 we take $k=\frac{2}{3}$ as a reasonable but somewhat arbitrarily chosen exponent $^{*}$. For simplicity we take $\sigma=\sigma_{1}$ (to be constant). The nodal movement can be expressed in terms of the function $h$ by substituting the time variable in (5.15):

$$
h=\sigma t^{\frac{2}{3}} \Longrightarrow t=(h / \sigma)^{3 / 2} .
$$

Substituting (5.17) into (5.15) yields $x=X(0)\left(1+\sigma^{1 / 4} h^{3 / 4}\right)$.

In summary, the equations (5.4), (5.5) and (5.12), (5.13) together with (5.15), (5.16) define the equations on the growing domain and the growth functions respectively.

\section{One-dimensional Growth Patterns}

To analyse the patterning properties of this model on a growing domain we must first select parameter values. As our model is hypothetical, we cannot use explicit

\footnotetext{
${ }^{\star}$ Different values of $k$ have been used to obtain similar results.
} 
Table 1. The range of parameter values for $\gamma$ and corresponding excited wavenumbers in one dimension for the Schnakenberg model: parameter values are $a=0.1, b=0.9$ and $d=10$ (see text for details).

\begin{tabular}{cc}
\hline $\begin{array}{c}\text { Range of values } \\
\text { of } \gamma\end{array}$ & $\begin{array}{c}\text { Wavenumbers } \\
\text { excited }\end{array}$ \\
\hline $0-19$ & - \\
$20-49$ & $k_{1}=\pi$ \\
$50-78$ & - \\
$79-177$ & $k_{2}=2 \pi$ \\
$178-197$ & $k_{2}=2 \pi, k_{3}=3 \pi$ \\
$198-315$ & $k_{3}=3 \pi$ \\
$316-444$ & $k_{3}=3 \pi, k_{4}=4 \pi$ \\
$445-493$ & $k_{4}=4 \pi$ \\
$494-710$ & $k_{4}=4 \pi, k_{5}=5 \pi$ \\
$711-789$ & $k_{4}=4 \pi, k_{5}=5 \pi, k_{6}=6 \pi$ \\
\hline
\end{tabular}

experimental data to choose parameter values. We assume that the growth of the ligamental attachment area occurs on the time scale of days as observed. For reasons of computational efficiency and running time, our $\sigma$ we have taken is a bit large. Typical chemical reactions occur in seconds. Arcoid ligaments range from $2-3 \mathrm{~mm}$ to $50 \mathrm{~mm}$ or more in length and from $2-3 \mathrm{~mm}$ to $10 \mathrm{~mm}$ in height. A typical glycymerid ligament might be $25 \mathrm{~mm}$ long and $6 \mathrm{~mm}$ in height. Therefore we assume that the length scale $L_{x}$ is of the order of $\mathrm{mm}$. The diffusion coefficients of the morphogens are of the order $10^{-8}-10^{-10} \mathrm{~cm}^{2} \mathrm{~s}^{-1}$. In all the simulations, we fix the values $a=0.1$ and $b=0.9$ and use standard linear stability analysis (see Appendix A) to choose $\gamma$ and $d$ such that the homogeneous steady state $\left(u_{s}, v_{s}\right)$ is linearly unstable in the presence of diffusion of the reactants. In one dimension, we fix the value of $d=10$ and then increase $\gamma$ until the uniform steady state becomes unstable (see Table 1 for the precise values). Convergence to numerical solutions is required to an accuracy of $10^{-8}$.

Now we are in a position to simulate changes in the spatial pattern of chemical concentrations as the initial non-dimensional domain $[-1,1]$ expands over time, representing the allometric growth of the ligamental area. We illustrate our results by two types of plots for each $\gamma$ : in each figure (Figs 8-10), the $x-t$ and $x-h$ plots [(a), (c) and (b), (d) respectively] correspond to the same numerical computation. The first plot shows the growth rate function of the domain. The second plot shows the height of the ligamental attachment area plotted against domain growth. In all the simulations, the height is given by the expression $h=\left(\frac{\frac{x}{X(0)}-1}{\sigma^{1 / 4}}\right)^{4 / 3}$. Numerical solutions are plotted at every 500 time-steps.

We explore the effects of various boundary conditions on the output of our model, anticipating that these will play an important part in defining growth patterns that 
(a)
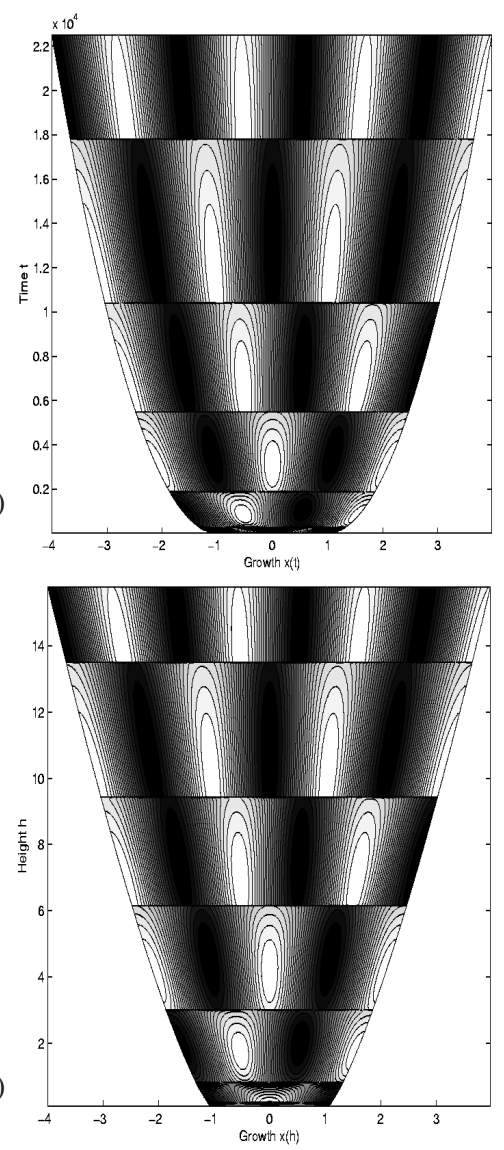

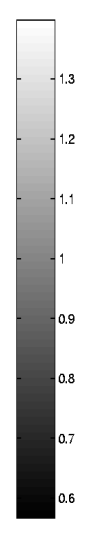

(b)
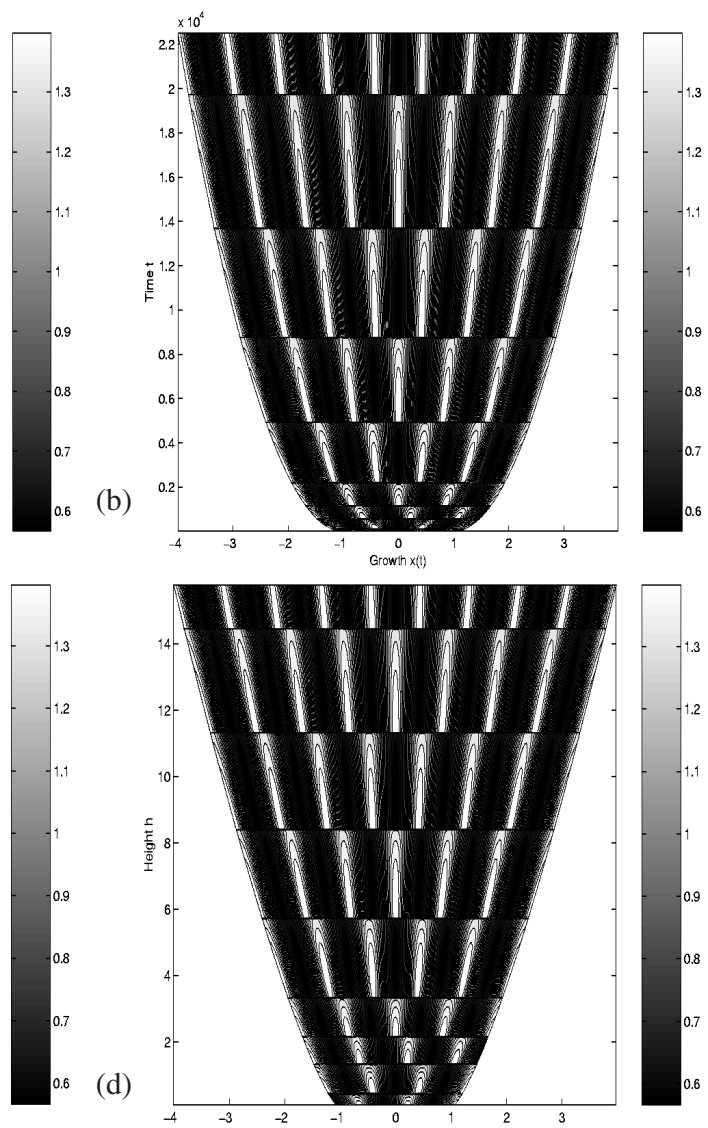

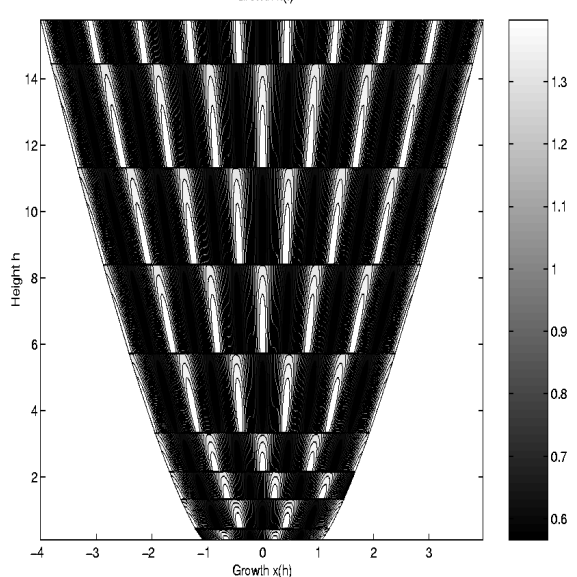

Figure 8. Numerical results of patterns of component $u$ on the growing $x$-domain, displayed in a $x(t)-t$ or $x(h)-h$ coordinate system, respectively, [for two values of $\gamma$ : $\gamma=29$ in (a), (c) and 185 in (b), (d)] of the reaction-diffusion equations (4.1) and (4.2) with homogeneous Neumann boundary conditions at the end points. Parameter values used are $a=0.1, b=0.9$ and $d=10$. The interval $[-1,1]$ is grown until it reaches the final interval $[-4,4]$. For the case $\gamma=29$ [(a) and (c)]; there are two length intervals in which there is no excitable spatially inhomogeneous solution. These numerical results confirm linear stability theory (Appendix A) in one dimension. With these types of boundary conditions, the results do not simulate any of the observed growth patterns of the arcoids nor the noetiid ligaments.

must conform to the size and shape of the expanding attachment area of the ligament. Recall that in typical arcoids, alternating sheets of lamellar and fibrous material run parallel to the anterior and posterior margins of the growth domain, and new elements are added at the centre of the pattern. In contrast, the patterns of noetiid ligaments are oriented perpendicular to the hinge axis, parallel to the direction of growth, and here new elements are added at the anterior and posterior margins. We predicted that this difference in growth patterns could be controlled by a relatively simple change in boundary conditions. 
(a)
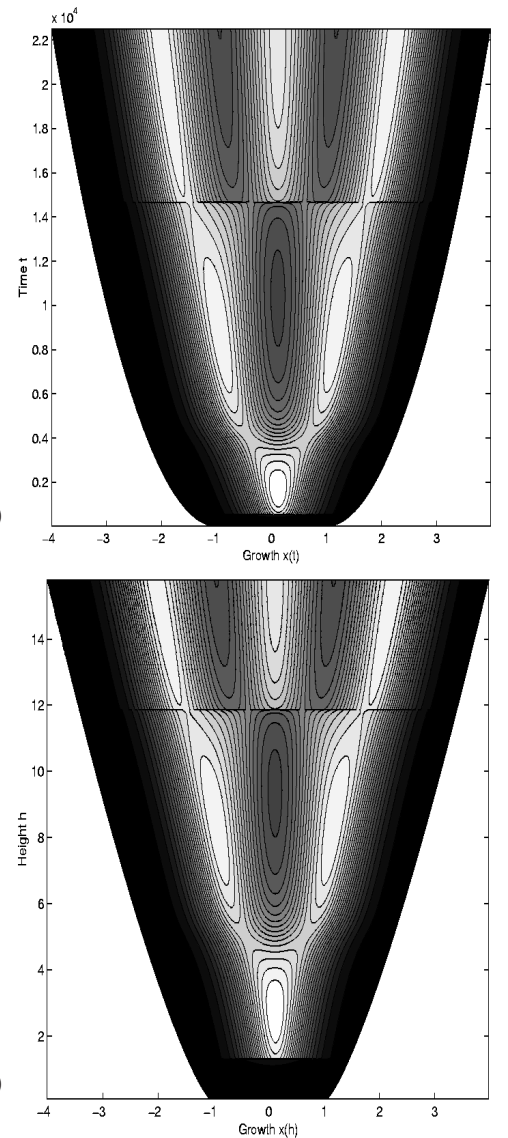

(b)
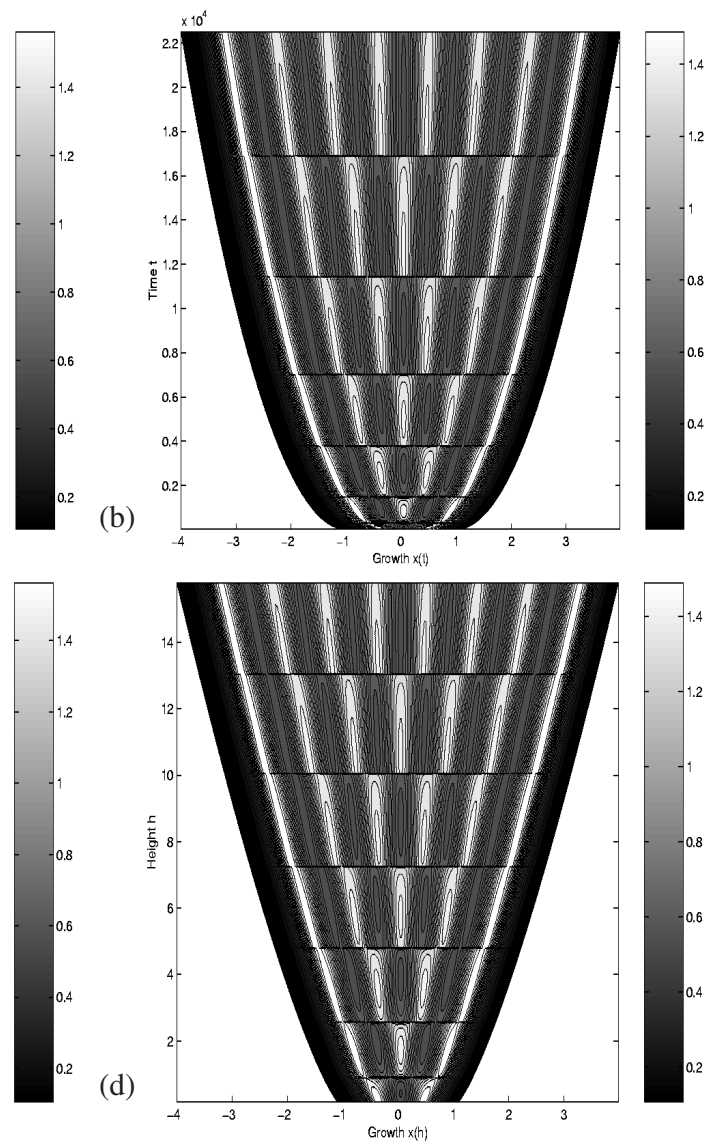

Figure 9. Numerical solution of patterns of component $u$ on the growing $x$-domain, displayed in a $x(t)-t$ or $x(h)-h$ coordinate system, respectively, of the reaction-diffusion system (4.1) and (4.2) with fixed boundary conditions on $v(v=0.5)$ and homogeneous Neumann boundary conditions on $u$. Parameter values as in Fig. 8. The numerical results are consistent with the growth patterns of Limopsis and the juvenile of any arcoid with a duplivincular ligament [compare (c) with Fig. 1(d)] or Glycymeris [compare (d) with Fig. 1(a)].

Let us first consider homogeneous Neumann boundary conditions. Linear stability analysis (Appendix A) predicts that there exist certain ranges of values of $\gamma$ where no spatially inhomogeneous solution is excited (Table 1). Figure 8 illustrates the corresponding numerical computations with homogeneous Neumann boundary conditions for different values of $\gamma$. In all the numerical results we only plot $u$ (the spatial profiles of $v$ are $180^{\circ}$ out of phase with those of $u$ ). The evolution of these patterns depicts two key factors. The solutions converge to a homogeneous steady state if no inhomogeneous solution is admissible. This is seen in Fig. 8(c) where initially no heterogeneous spatial pattern is exhibited. The second key factor is that solutions are not symmetric in general [Fig. 8(d)]. Hence these simulations for the system (4.1) and (4.2) on a growing domain confirm results from linear stability 
(a)

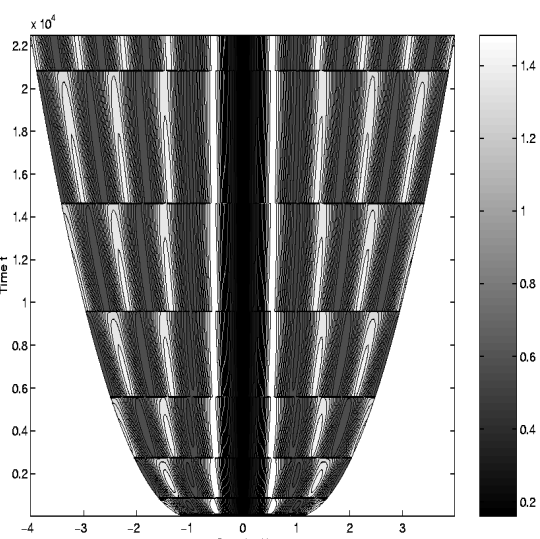

(c)

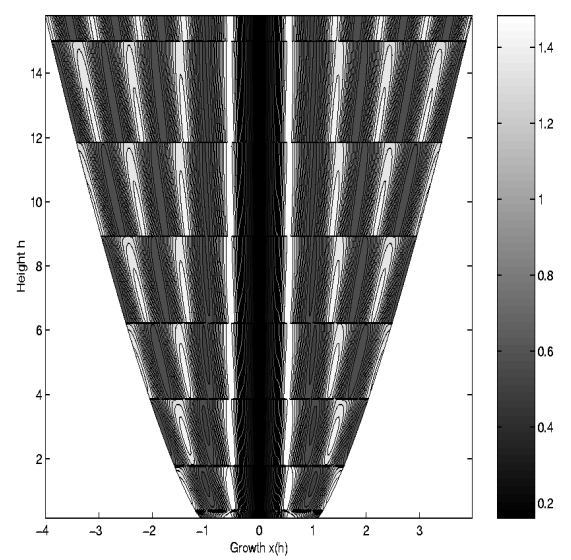

(b)

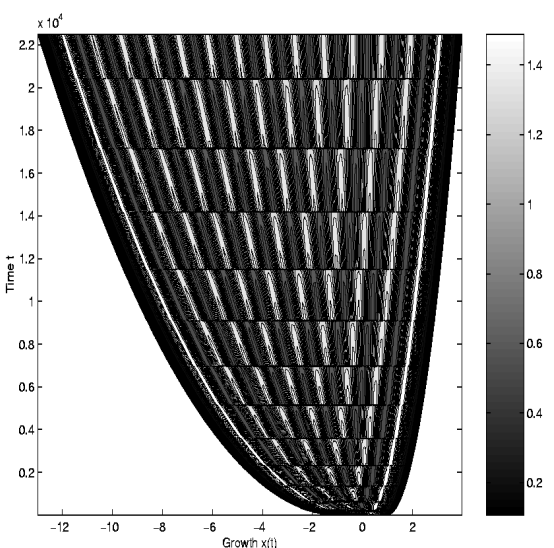

(d)

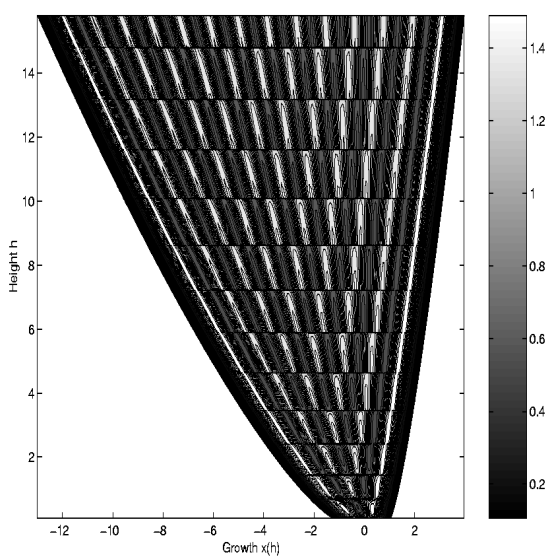

Figure 10. (a), (c) The numerical solution of patterns of component $u$ on the growing $x$-domain, displayed in a $x(t)-t$ or $x(h)-h$ coordinate system, respectively, for equations (4.1) and (4.2) when an internal boundary condition, $v(0)=0.5$ is imposed, together with homogeneous Neumann boundary conditions for both reactants at the end points $(\gamma=185)$. Parameter values as in Fig. 8. The results are consistent with the patterns observed in noetiid ligament growth (cf. Fig. 5). The results in (b) and (d) $(\gamma=114)$ are computed for non-uniform growth functions with homogeneous Neumann boundary conditions for both species at the domain end points (no internal boundary condition). The growth rate of the interval $[-1,0]$ is four times the value of $\sigma$ in the interval $[0,1]$. These simulations exhibit an intermediate growth pattern, where strips of lamellar ligament initially run almost parallel to the margin of the attachment area, then turn to run perpendicular to the hinge axis (compare with patterns in Fig. 5).

analysis which were only derived on fixed domains. However, these computed results do not simulate typical observed arcoid ligament growth patterns. Observations of the growth patterns of living and fossil arcoids indicate that lamellar and fibrous materials are added continuously to their ligaments, above the scale of tidal or daily increments. Therefore, we see that reaction-diffusion theory in which the domain boundary is considered impermeable to the reactants is unable to exhibit the type of spatial pattern sequence observed during arcoid or noetiid ligament morphogenesis. 
We now assume mixed boundary conditions of the following type, $v=0.5$ and $u_{n}=0$ along the end points of the interval. That is, we impose fixed boundary conditions on chemical concentration $v$ and homogeneous Neumann boundary conditions on $u$. Figure 9 illustrates the numerical solution for these mixed boundary conditions. Note that these solutions are symmetric and they are always spatially inhomogeneous. With these boundary conditions, the model simulates the symmetrical, chevron growth pattern of typical arcoid ligaments. For $\gamma=29$, a growth pattern with a single pair of marginal sheets of lamellar ligament is represented in Fig. 9(a) and 9(c). This corresponds to the ligaments of most juvenile arcoids and Limopsis [Fig. 1(c) and 1(d)]. With $\gamma=185$, the model simulates multiple; symmetrical alternating sheets of lamellar and fibrous ligament material, disposed at an oblique angle to the hinge axis [Fig. 9(b) and 9(d)]. This chevron pattern, known as the duplivincular ligament [Fig. 1(a)], is characteristic of Glycymeris and many other arcoids. The numerical results exhibit oblique patterns which run parallel to the margin of the ligament attachment area. It must be remarked here that the numerical results are robust under small changes in the boundary values for $v$ (see Section 8 for more details).

The growth patterns of noetiid ligaments can be simulated by imposing an internal boundary condition $v=0.5$ at $x=0$ and homogeneous Neumann boundary conditions for both reactants at the external boundaries [Fig. 10(a) and 10(c)]. Physically, the internal boundary condition models the insertion of vertical strips of lamellar ligament material by keeping the site of insertion of the central strip fixed. The growth of the ligamental area creates space for the insertion of new lamellar elements at the distal margins of the ligament [Fig. 1(f)]. The pattern simulating alternating lamellar and fibrous elements runs perpendicular to the hinge axis, as in the ligament pattern of noetiids.

To compute growth patterns that simulate the transition from one or more oblique lamellar chevrons running parallel to the margin of the ligament, to vertical strips we assume that the growth rate of the subinterval $[-1,0]$ is four times ${ }^{\S}$ the growth rate of the subinterval $[0,1]$. Boundary conditions are the same as those considered to simulate Limopsis. Figure 10(b) and 10(d) illustrate numerical results under different growth rates of the ligament, these exhibit growth patterns consistent with those seen in the ontogeny of L. marionensis and in the early growth stages of the noetiid ligament in some species of Striarca (Fig. 5).

\section{Two-dimensional Growth Patterns}

Although ligament patterning in arcoid bivalves is essentially due to pattern formation on a 1D domain, it is interesting from a mathematical point of view to investigate whether the above results can be extended to $2 \mathrm{D}$ domains. For illustrative

\footnotetext{
$\S$ This factor is not significant. We have also computed with various factors $(1.5,2.0,2.5,3.5$ and 4.5) to obtain similar patterns.
} 
purposes, we consider an isosceles triangle with vertices $(0,0),(-2,-1)$, and $(2,-1)$ [Fig. 11(a)]. This triangle has an aspect ratio of $2: 1$ which compares with the actual ratio in the bivalve (see Section 6). The triangle is grown vertically downwards as illustrated in Fig. 6 with growth function given by (5.16). To simulate oblique chevron patterns we assume the same boundary conditions as those in one dimension given as follows: along the oblique sides $v=0.5$ and along the horizontal side $v_{n}=0 ; u_{n}=0$ along all three sides. Figure 11 shows numerical results with these boundary conditions, initial conditions are taken as random perturbations about the uniform steady state.

As the triangle grows vertically downwards oblique chevron patterns are obtained as a result of peak splitting and peak insertion (see Fig. 11). Observe that oblique patterns along the oblique sides do not change with domain growth but remain fixed. Once oblique patterns are established, these remain fixed with the exception of those that are more or less in the middle of the horizontal side, which deform accordingly. Unlike in one dimension, where the solutions at each time are 'packed' one on top of the other in a 1D fashion, here, growth patterns emerge as the whole triangular domain grows vertically downwards. Surprisingly though, the patterns exhibited are qualitatively similar to those obtained in one dimension.

In order to simulate $2 \mathrm{D}$ vertical strips with the same boundary conditions as those shown in Fig. 10(a) and 10(c) we observe that these patterns are symmetrical along the line $x=0$. Therefore we consider numerical simulations on a triangle with vertices $(0,-1),(0,0)$ and $(-2,-1)$ and then use symmetry to obtain the patterns on the whole triangular domain given as before by vertices $(0,0),(-2,-1)$ and $(2,-1)$. Figure 12 shows vertical strips exhibited as the domain grows vertically downwards. These patterns compare qualitatively with those obtained in Fig. 10 (a) and 10(c) for the 1D case. It can be observed that once the vertical strips are established, these are fixed and no changes occur in terms of their location and scale. New strips emerge at the end-points of the horizontal edge. The 2D pattern formation of vertical strips differs from the 1D case where peak splitting and peak insertion take place along the whole horizontal $x$-axis as opposed to the end points only.

Qualitatively, the numerical simulations are independent of the dimensionality. In both the one- and 2D domains we have carried out numerical computations with the same boundary conditions. The results on growing triangular domains are the same as those obtained on a growing 1D interval. In two dimensions, the oblique chevron patterns are formed as a result of peak insertion along the middle of the horizontal side of the growing triangle. On the other hand, vertical strips are formed through peak splitting and peak insertion along the end points of the horizontal side. These simulations show that the mechanism by which the pattern increases in complexity as the domain grows is independent of spatial dimension in this case.

For mixed boundary conditions, the results on the large final fixed domain are the same as those eventually observed on taking a small triangle and growing it to the large triangle. However, if Neumann boundary conditions are applied, the patterns 
(a)
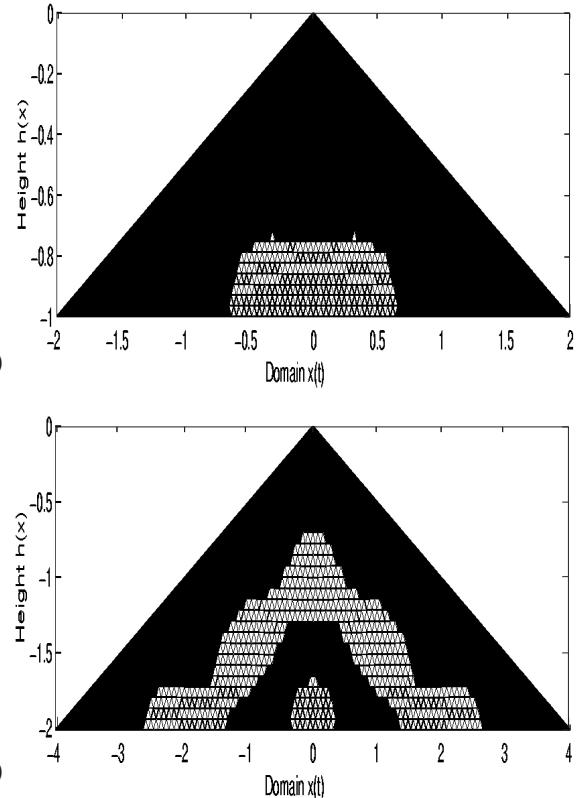

(c)

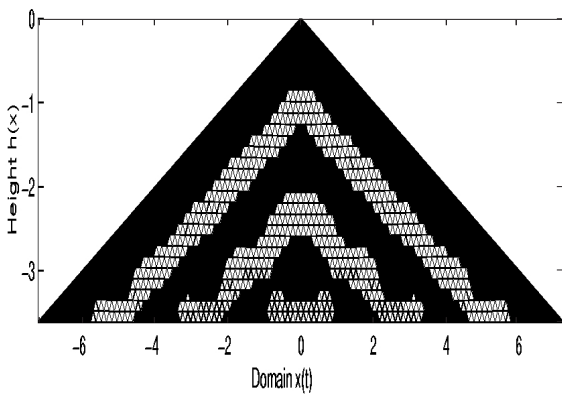

(b)

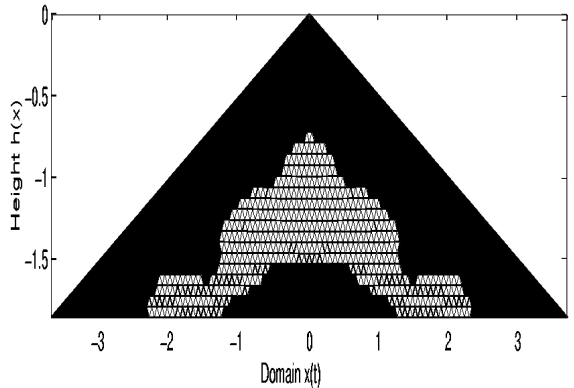

(d)
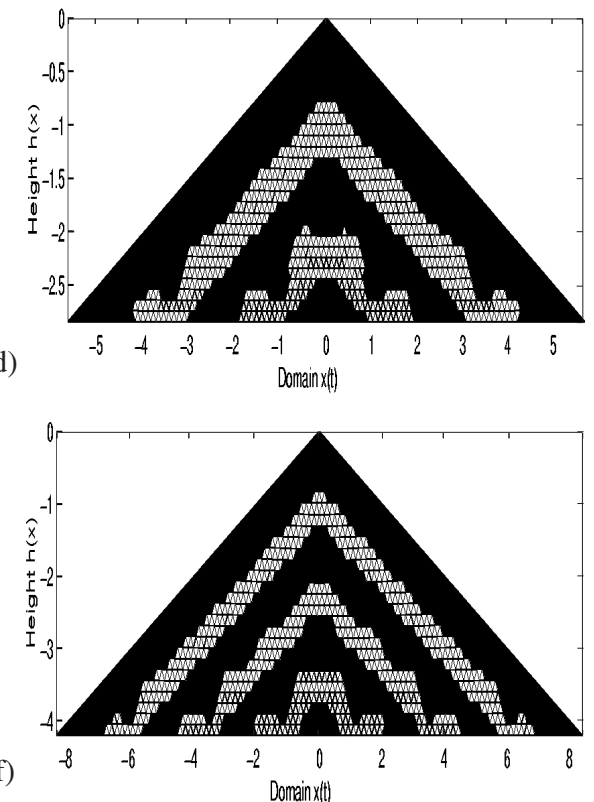

Figure 11. Simulations of patterns of component $u$ on the growing $x$-domain, displayed in a $x(h)-h$ coordinate system, obtained with the Schnakenberg model with parameter values $a=0.1, b=0.9, d=10$ and $\gamma=114$. The simulations are carried out on an unstructured mesh of 2260 elements and 1211 nodes. This mesh is generated automatically using a Delaunay triangulation (Müller et al., 1993). These simulations compare qualitatively with the 1D results shown in Fig. 9(c) and 9(d). We shade according to the following procedure: for each triangular element, we calculate the minimum (or maximum) value of the three nodal values of the numerical solution at the three vertices of this element. Given a constant threshold value, if this value is less than the minimum value, say, shade this element black, otherwise shade it white. Similarly when the maximum value is used in place of the minimum value. In all our simulations, the constant threshold value is taken as 0.9 for illustrative purposes. Therefore shading is done element-wise rather than globally. Biologically; we can think of one of the chemicals in the model as causing cells to differentiate if its concentration lies above a threshold level. Hence the black-shaded cells would exhibit a differential fate different from that of the white-shaded cells. 
(a)
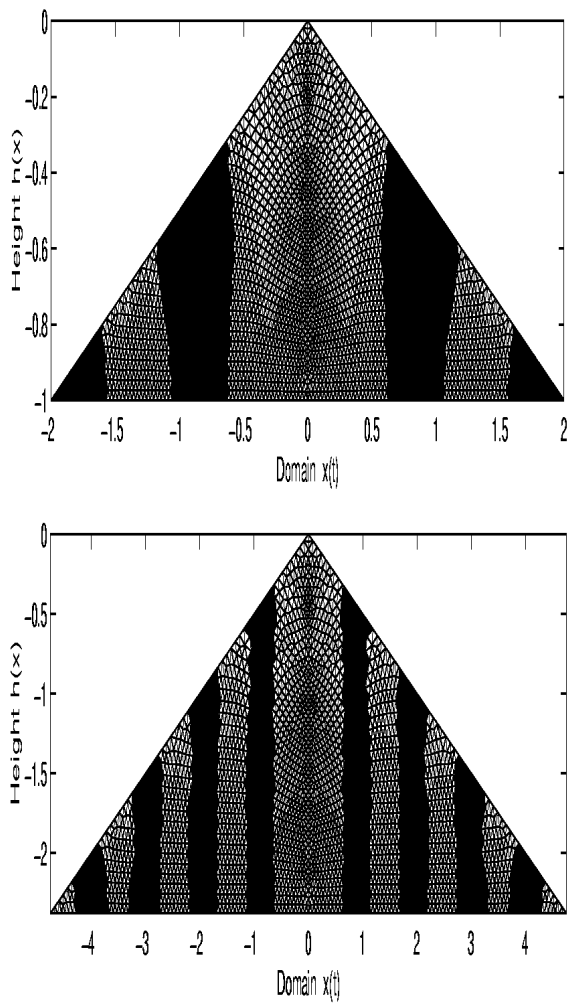

(c)

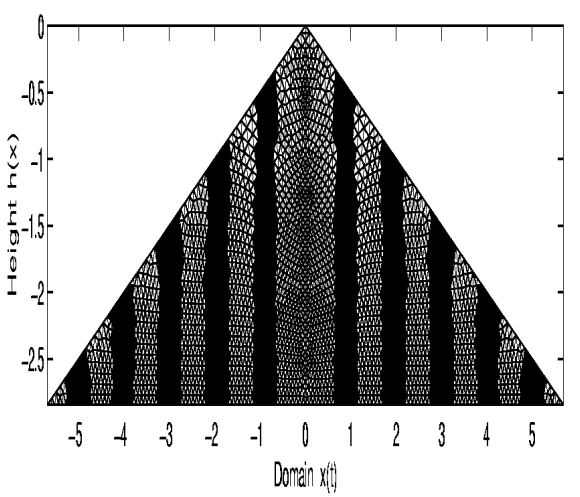

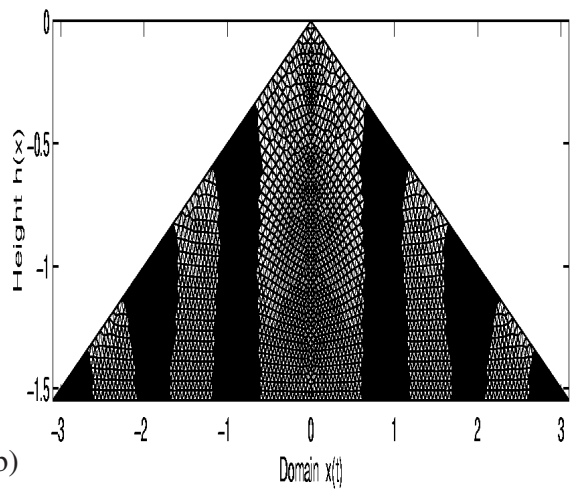

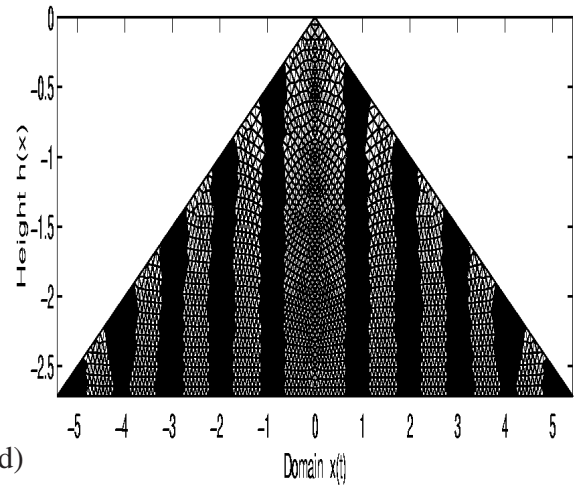

(d)

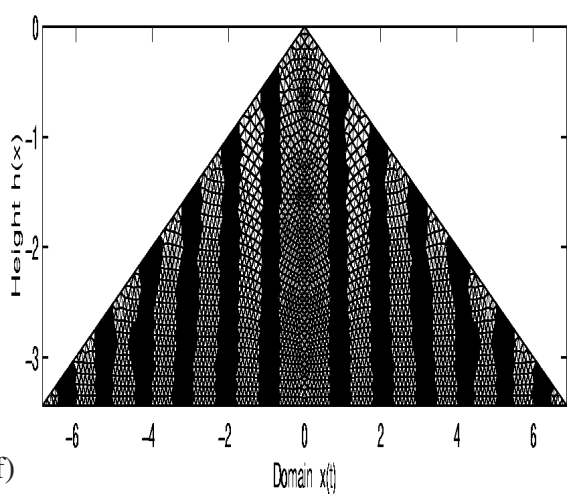

Figure 12. Numerical simulations of patterns of component $u$ on the growing $x$-domain, displayed in a $x(h)-h$ coordinate system, of the Schnakenberg reaction model (4.1) and (4.2) with boundary conditions $v=0.5$ along $x=0$ and Neumann boundary conditions for both reactants elsewhere along the boundary. Parameter values as in Fig. 11. Vertical stripe patterns are exhibited. These patterns are qualitatively similar to those obtained in one dimension [compare with Fig. 10(a) and 10(c)].

observed on the final fixed triangle are substantially different from those obtained under domain growth (Madzvamuse, 2000). Hence growth alone is not enough to generate chevron patterns; appropriate mixed boundary conditions are also crucial. 
(a)

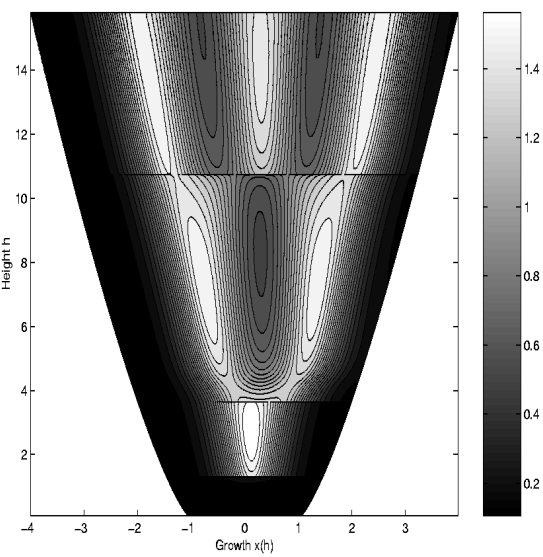

(c)

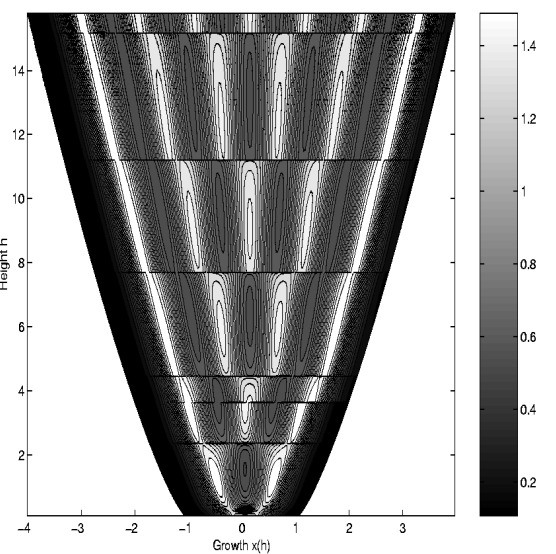

(b)

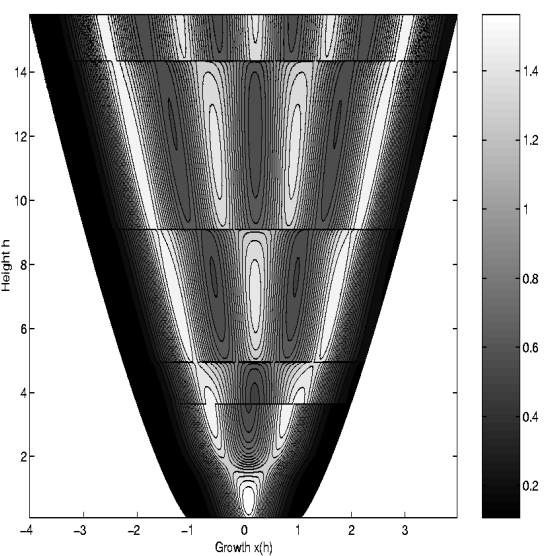

(d)

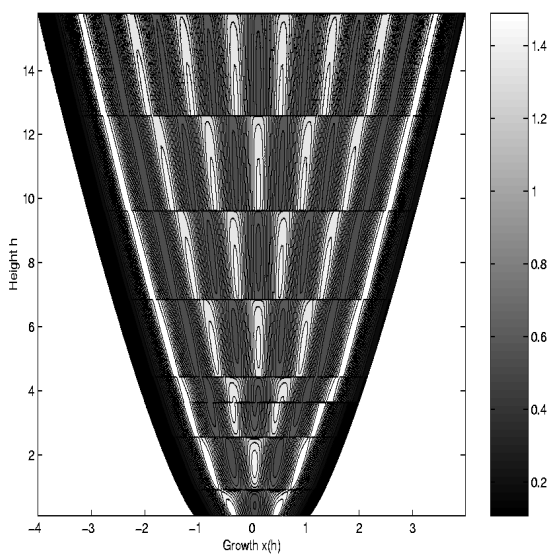

Figure 13. Numerical simulations of patterns of component $u$ on the growing $x$-domain, displayed in a $x(h)-h$ coordinate system, of equations (4.1) and (4.2) showing the effects of changing external boundary values. The parameter values are $a=0.1, b=0.9, d=10$ and (a) $\gamma=29$, (b) $\gamma=60$, (c) $\gamma=114$, (d) $\gamma=185$. Here, $u_{n}=0, v=0.5$ as the domain grows from $[-1,1]$ to $[-2,2]$ and $u_{n}=0, v=1.0$ as the domain grows from $[-2,2]$ to $[-4,4]$. The results compare qualitatively with those shown in Fig. 9.

\section{Experimental Tests and Predictions}

In this section we present $1 \mathrm{D}$ experimental tests and predictions that our mathematical model is capable of generating. We present in Fig. 13 the effects of changing external boundary values. The boundary values of one of the chemical concentrations, $v$, say, is changed from 0.5 when the domain reaches twice its original size to 1.0. From these simulations we see that changes in external boundary values have no major effect on simulated patterns. This is significant in that the patterns obtained are independent of boundary values. Similarly, changes in the internal boundary values have no effect on the simulated patterns, as illustrated in Figs 15 and 16. For example, in Fig. 16 the values of the chemical concentration $v$ are randomly generated along the internal boundary. Therefore we predict that the 
(a)

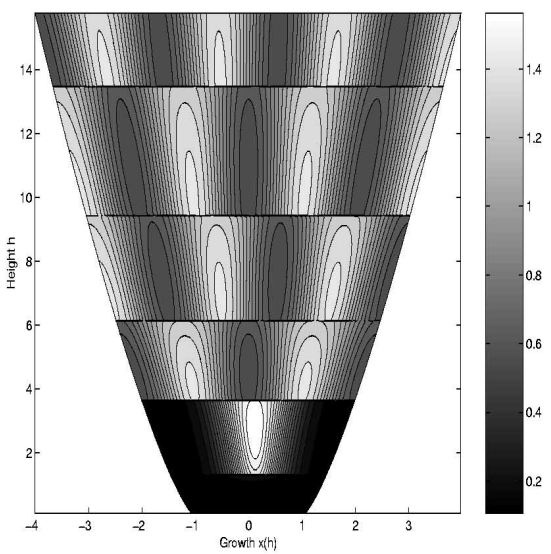

(c)

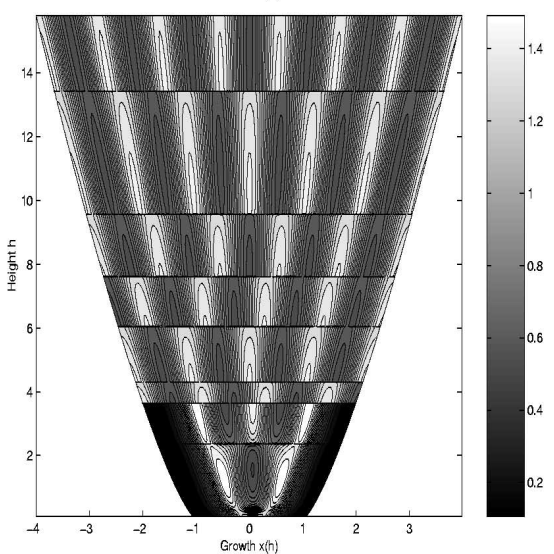

(b)

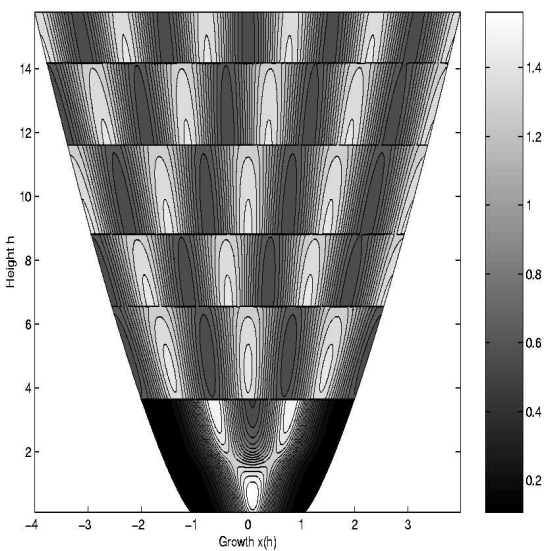

(d)

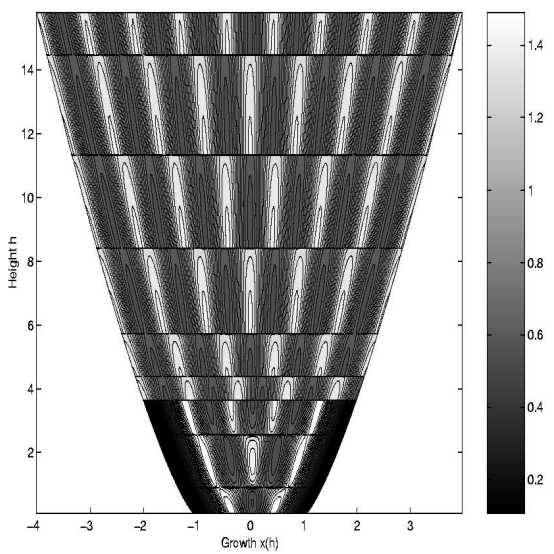

Figure 14. Simulations of patterns of component $u$ on the growing $x$-domain, displayed in a $x(h)-h$ coordinate system, showing the effects of changing the nature of external boundary conditions, a change from mixed boundary conditions to zero-flux (Neumann) boundary conditions. Parameter values as in Fig. 13. $v$ is fixed initially to 0.5 along $x=-1$ and 1 , until the domain reaches twice its original size, and then is set to zero-flux for the remainder of the growth. Clearly, there is a dramatic change in pattern when the external boundary conditions are changed from mixed to zero-flux.

quantity of the source along the oblique sides or along the centre of the domain does not influence the patterns exhibited and hence growth patterns are robust to changes in boundary values.

However, a complete change in the nature of the boundary conditions affects simulated growth patterns. For example, if we allow a change from mixed boundary conditions to zero-flux (Neumann) boundary conditions substantial changes occur. Figure 14 shows the effects of such changes. In this case, a change from prescribing a source along the distal oblique margins to a self-organization process brings about a complete change in growth patterns. 
(a)

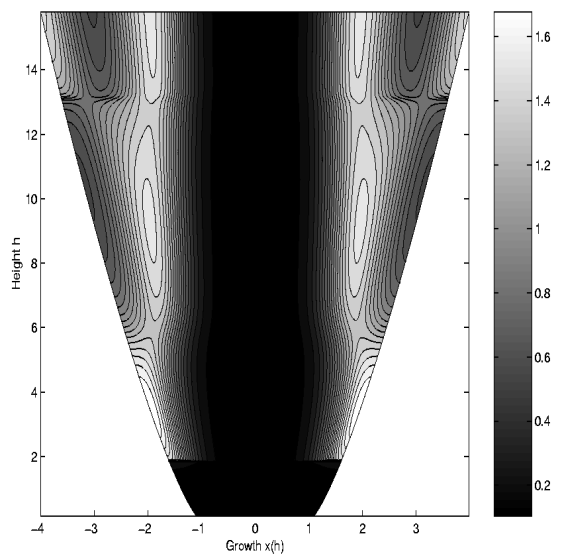

(c)

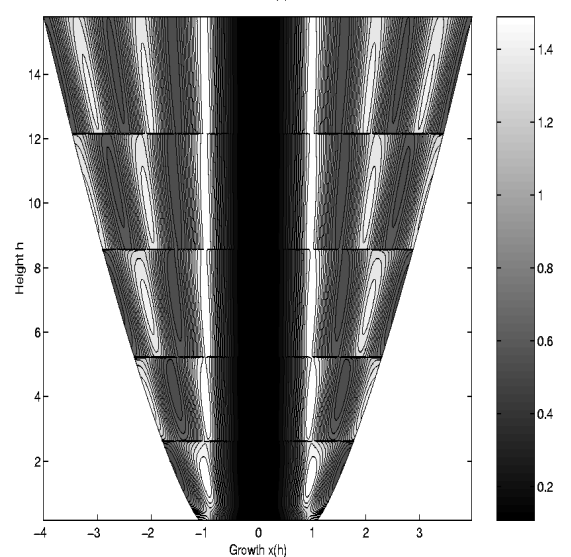

(b)

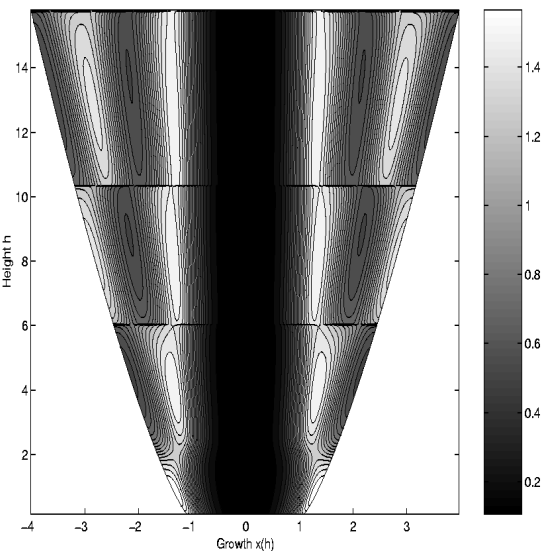

(d)

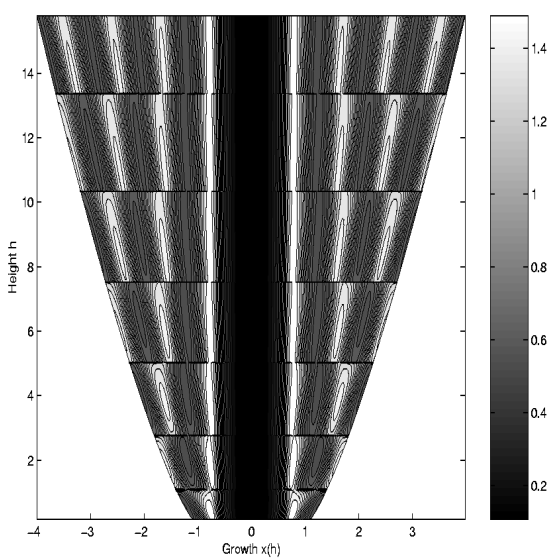

Figure 15. The effects of changing internal boundary values on numerical simulations of patterns of component $u$ on the growing $x$-domain, displayed in a $x(h)-h$ coordinate system. Parameter values as in Fig. 13; $v=0$ along $x=0$ and $u_{n}=v_{n}=0$ along $x=-1$ and $x=1$. Patterns simulated remain qualitatively similar to those shown in Fig. 10(a) and 10(c).

\section{Discussion AND CONCLUSION}

We have simulated the growth patterns of arcoid ligaments, showing how a fairly simple reaction-diffusion model can generate this wide range of patterns under the control of very few conditions and parameters, on a growing domain.

Specifically, it has been shown that the spatial patterns exhibited by a reactiondiffusion system on a growing domain are consistent with the patterns of ligaments observed in nature. For example, we have computed typical arcoid ligament growth patterns of Glycymeris. The addition of new elements at or near the middle of the growth zone, as the ligament expands along the hinge axis, has been achieved by considering a general reaction-diffusion system with specific boundary conditions. Typical noetiid ligament growth patterns have also been computed. Here, new elements are added at the anterior and posterior ends of the ligament. A simple 
(a)

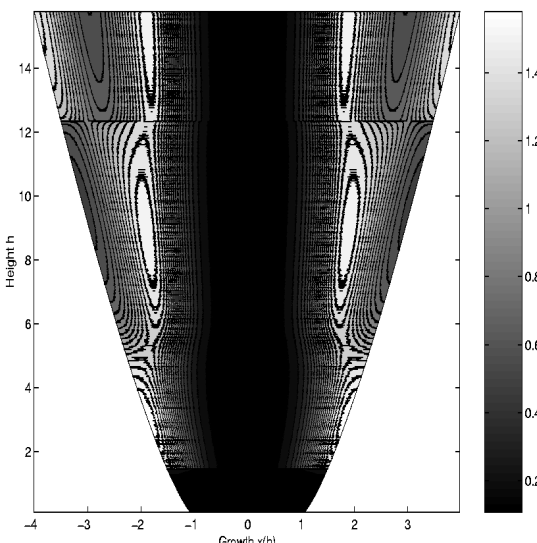

(c)

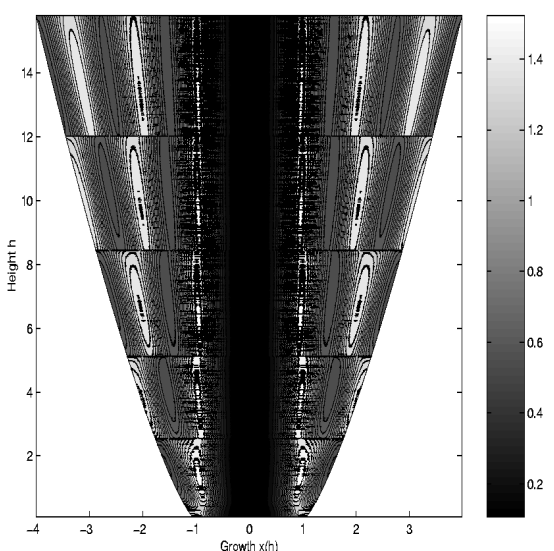

(b)

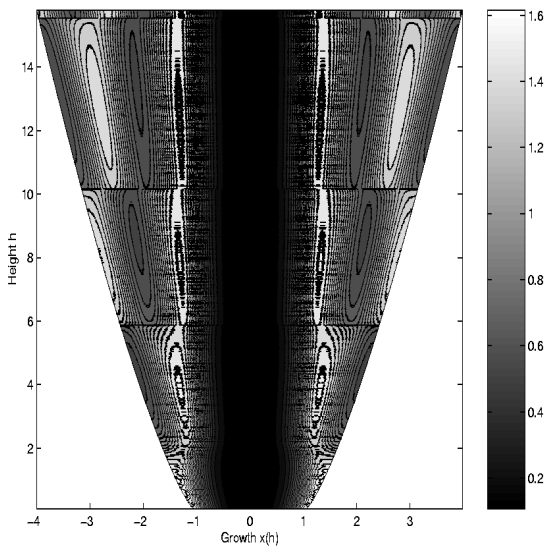

(d)

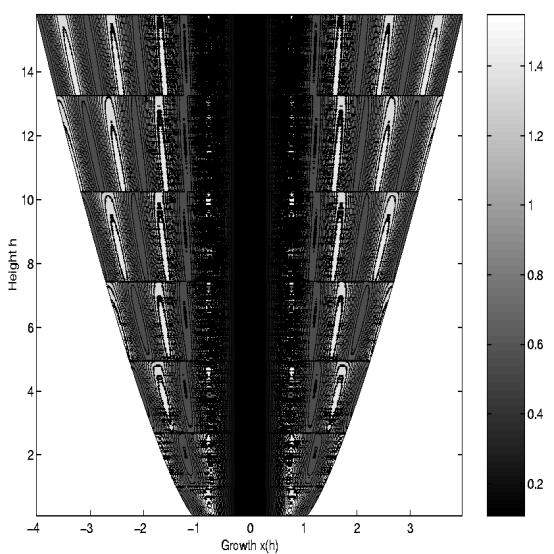

Figure 16. Parameter values are as in Fig. 13, but the fixed value of $v$ is randomly generated along $x=0$, and $u_{n}=v_{n}=0$ along $x=-1$ and $x=1$. The simulated patterns are qualitatively similar to those shown in Fig. 15.

change in boundary conditions is all that is required to generate this pattern, using the same general model that simulates the development of other arcoid ligaments.

Our study shows that what might have seemed to be rather fundamental differences in pattern, between the ligaments of typical arcoids and those of the noetiids, may be controlled by rather simple changes in the underlying developmental process. We have shown how the distinctive ligament of the noetiids is related, in terms of plausible underlying developmental processes, to the duplivincular ligament of more typical arcoids from which it evolved. To shift the site where new elements are added to the pattern, from near the midpoint of the ligament to its extremities, only a change in the boundary conditions of the morphogenetic field is required. This change causes the space created by growth of the ligament for insertion of new sheets of lamellar ligament to open up at the distal margins of the ligament, rather than at the middle.

In Limopsis, for example, the normal growth pattern yields a single pair of oblique, lamellar sheets, with medial growth of an expanding wedge of fibrous 
ligament. To obtain vertical strips in our model, we simply imposed an internal boundary condition. To account for the transformation from an oblique pattern to the pattern of vertical strips, we considered different growth rates of the domain in which the ligament develops [Fig. 10(b) and 10(d)]. Without making any other change in the system, we were able to simulate the observed variations. We have shown here that different developmental models are not required to simulate both the oblique growth patterns of limopsid and arcoid ligaments and the vertical patterns of the noetiids. Likewise, the aberrant variants observed in L. marionensis can be simulated by a reaction-diffusion system set up to simulate the glycymerid ligament, with appropriate changes in boundary conditions.

These results have potentially important implications for our understanding of evolutionary relationships among the arcoid bivalves. The fact that their ligaments can be simulated by a common developmental program underscores the unity of the Arcoida, which is well established on other grounds. On the other hand, the Noetiidae, which has long been recognized as a distinct family (MacNeil, 1937; Newell, 1969) may prove not to be a cohesive group. The presence of a ligament composed of vertical strips, as opposed to oblique, duplivincular patterns, is the only character these animals all share. In several respects, including the size, shape and disposition of the ligament, the noetiids are quite heterogeneous. If the noetiid pattern can easily be derived from the general arcoid pattern, it may have arisen more than once, in which case it would not be a reliable indicator of common ancestry.

Our model accounts for five of the six patterns illustrated in Fig. 1. A subsidiary repetition of the main growth pattern appears at one end of a Limopsis ligament, illustrated in Fig. 1(e). This secondary element in the growth pattern began to develop at the same time as another small pair of lamellar sheets within the main growth pattern. This suggests that a new patterning domain was established simultaneously with the initiation of a new element within the main domain, presumably in response to some common influence on the growth and function of the ligament. Note that the pattern in the large domain is similar to that in Fig. 1(a), but that symmetry is not preserved and the spacing of the lamellar sheets is irregular.

Deviations from the regular pattern of the ligament, such as that just noted, are common in most species of Arca and in relatively large forms of Limopsis. These 'mistakes' in the growth process give rise to patterns with a local, stochastic component, like most of the shell colour patterns modeled by Meinhardt (1995). Thus, in certain circumstances, the boundary constraints set by the shape of the ligamental attachment area are relaxed. When this occurs, the system reverts to purely local control of activation and inhibition by the morphogens. This merits further study, as it bears on the underlying mechanisms by which the secretion of lamellar and fibrous ligament are controlled.

The implementation of the moving finite element method has been carried out on a moving mesh whose movement has been specified. This is a novel use of the moving grid finite element method and differs substantially from its traditional 
implementation, where both the nodal values of the grid movement and the solution values are assumed unknown and therefore have to be calculated simultaneously (Baines, 1994). The generality of the application of the moving finite element method on growing domain problems may possibly allow us to investigate a wide variety of growth and patterning problems in biological applications.

The numerical solutions are robust to small changes in the values of the boundary conditions. Not only that, they are also robust to domain growth in the sense that the sequence of patterns observed is independent of the initial conditions. Similar patterns can be produced by different reaction-diffusion models, such as the Thomas (1975) and Geirer and Meinhardt (1972) models, as has been confirmed by numerical simulations.

Different growth rate functions $r(t)$ have been used in the numerical computations (results not shown here) to produce qualitatively similar patterns as those reported in this paper. Not only that, the magnitude of $\sigma$ becomes an issue only if it is taken very large. Taking smaller values has no effect on the qualitative nature of the patterns, only that the computation time taken for the growing domain to reach its final size grows exponentially with smaller values of $\sigma$.

We have shown that under certain circumstances the growth patterns of a reaction-diffusion system on a $1 \mathrm{D}$ growing domain are qualitatively similar to those obtained on a growing triangle. In both cases, domain growth and boundary conditions are shown to be the major factors in selecting patterns. However, pattern selection in two dimensions occurs on the whole domain as it grows, unlike the $1 \mathrm{D}$ case.

We have shown how changing boundary conditions can lead the model to exhibit a fundamental change in behaviour from simple peak insertion (Fig. 12) to peak splitting and insertion [Fig. 11(b)]. Note that a similar change could be achieved by keeping the boundary conditions fixed and changing the kinetics. For example, Crampin et al. (2001) have shown that, on a 1D domain, the presence of a quadratic nonlinearity will lead to mode doubling via peak splitting or insertion (depending on the exact form of the nonlinearity), while a purely cubic nonlinearity leads to mode tripling. Note that saturating kinetics can also give insertion/splitting depending on the level of saturation (Meinhardt, 1995).

We have also shown how the model can be used to make experimentally testable predictions on disruption of the boundary of the growing ligament.

\section{ACKNOWLEDGEMENTS}

This work was supported by National University of Science and Technology of Zimbabwe (AM), Franklin \& Marshall College (RDKT), and an EPSRC grant (GR/R03914) awarded to AJW and PKM. We would like to thank an anonymous referee for several very helpful suggestions. 


\section{APPENDix A: Linear ANALYSIS}

Standard linear stability analysis shows that diffusion-driven instability of a steady state of the system (4.1) and (4.2) occurs if the following conditions hold [see, for example, the books by Edelstein-Keshet (1988) and Murray (1993)]

$$
\begin{aligned}
f_{u}+g_{v} & <0, \\
f_{u} g_{v}-f_{v} g_{u} & >0, \\
d f_{u}+g_{v} & >0, \\
\left(d f_{u}+g_{v}\right)^{2}-4 d\left(f_{u} g_{v}-f_{v} g_{u}\right) & >0,
\end{aligned}
$$

where the partial derivatives are evaluated at the steady state. The inequalities (A1)-(A4) define a domain of parameter space, known as the Turing space, wherein the uniform steady state is unstable to random small perturbations.

Under these conditions, spatial disturbances with wavenumbers $k \in\left(k_{-}, k_{+}\right)$will initially grow, where

$$
k_{ \pm}^{2}=\gamma \frac{\left(d f_{u}+g_{v}\right) \pm \sqrt{\left(d f_{u}+g_{v}\right)^{2}-4 d\left(f_{u} g_{v}-f_{v} g_{u}\right)}}{2 d}
$$

are the roots of the function on the left-hand side of (A4). In the case we are interested in, namely, the unit interval with zero flux boundary conditions, a further restriction on $k$ is that it must take discrete values $n \pi$, corresponding to the spatial mode $\cos n \pi x$ denoted by $n$. For the Schnakenberg system (4.1) and (4.2) under zero flux boundary conditions, the steady state is given by $\left(a+b, \frac{b}{(a+b)^{2}}\right)$ To be specific, if we fix the values $a=0.1, b=0.9$ we have the steady state $(1.0,0.9)$.

For diffusion-driven instability to occur we require that the Turing conditions (A1)-(A4) are satisfied and that if we wish to isolate a certain mode, say $n$, then

$$
k_{n-1}^{2}<k_{-}^{2}<k_{n}^{2}<k_{+}^{2}<k_{n+1}^{2} .
$$

We wish to isolate a certain mode, that is, we want linear stability analysis to predict that the uniform steady state goes unstable only to spatial perturbations $\cos n \pi x$ with a particular $n$. We choose $d$ as a bifurcation parameter $(d=10)$ and vary $\gamma$ to isolate particular wavenumbers which may be excited. The table below shows the range of parameter values for $\gamma$ and the corresponding excited wavenumbers.

For example, in the range $\gamma \in[0,19]$ there does not exist an excitable wavenumber because the values of $\gamma$ are too small, so the homogeneous steady state is linearly stable. For the range $\gamma \in[20,49]$ there is only one wavenumber excited, namely $k_{1}=\pi$. On the other hand, in the range $\gamma \in[178,197]$, there are 
two modes to which the homogeneous steady state is unstable, namely those with wavenumbers $k_{2}=2 \pi$ and $k_{3}=3 \pi$ respectively. In this case the solution to the nonlinear model may depend on the initial conditions. If we wish to avoid multiple excitable solutions, for example, then we must not only vary $\gamma$ but also $d$.

\section{REFERENCES}

Baines, M. J. (1994). Moving Finite Elements, Monographs on Numerical Analysis, Oxford: Clarendon Press.

Baines, M. J. and A. J. Wathen (1988). Moving finite element methods for evolutionary problems. I. Theory. J. Comp. Phys. 79, 245-269.

Crampin, E. J. (2000). Reaction-diffusion patterns on growing domains. D Phil thesis, University of Oxford.

Crampin, E. J., E. A. Gaffney and P. K. Maini (2001). Mode-doubling and tripling in reaction-diffusion patterns on growing domains: a piecewise linear model. J. Math. Biol., in press.

Edelstein-Keshet, L. (1988). Mathematical Models in Biology, New York: Random House.

Ermentrout, B., J. Campbell and G. Oster (1986). A model for shell patterns based on neural activity. Veliger 28, 369-388.

Geirer, A. and H. Meinhardt (1972). A theory of biological pattern formation. Kybernetik 12, 30-39.

Jimack, P. K. and A. J. Wathen (1991). Temporal derivatives in the finite-element method on continuously deforming grids. SIAM J. Numer. Anal. 28, 990-1003.

Kiskaddon, L. (1996). Computer simulations of arcoid ligaments. Unpublished report on independent study, Franklin and Marshall College, Lancaster, Pa., 20p. + computer file.

Kondo, S. and R. Asai (1995). A reaction-diffusion wave on the skin of the marine angelfish. Pomacanthus, Nature 376, 765-768.

MacNeil, F. S. (1937). The systematic position of the pelecypod genus. Trinacria. J. Washington Acad. Sci. 27, 452-458.

Madzvamuse, A. (2000). A numerical approach to the study of spatial pattern formation. D Phil thesis, University of Oxford.

Meinhardt, H. and M. Klinger (1987). A model for pattern formation on the shells of molluscs. J. Theor. Biol. 126, 63-69.

Meinhardt, H. (1995). The Algorithmic Beauty of Sea Shells, Heidelberg, New York: Springer.

Morton, K. W. and D. F. Mayers (1994). Numerical Solution of Partial Differential Equations, Cambridge University Press.

Müller, J. D., P. L. Roe and H. Deconinck (1993). A frontal approach for internal node generation for Delaunay triangulations. Int. J. Numer. Methods Fluids 17, 241-256.

Murray, J. D. (1993). Mathematical Biology, Heidelberg, New York: Springer.

Newell, N. D. (1937). Late Paleozoic pelecypods: Pectinacea. Kansas Geological Survey, Publications 10, 1-123. 
Newell, N. D. (1969). Family Noetiidae Stewart, 1930, in Treatise on Invertebrate Paleontology, R. C. Moore, (Ed.) (Part N, Mollusca 6), Geological Society of America and University of Kansas, pp. 261-264.

Oster, G. F., N. Shubin, J. D. Murray and P. Alberch (1988). Evolution and morphogenetic rules: the shape of the vertebrate limb in ontogeny and phylogeny. Evolution 42, 862-884.

Painter, K. J. (1997). Chemotaxis as a mechanism for Morphogenesis. D Phil thesis, University of Oxford.

Reddy, J. N. (1984). An Introduction to the Finite Element Method, McGraw-Hill.

Saad, Y. (1996). Iterative Methods for Sparse Linear Systems, PWS Publishing Company.

Schnakenberg, J. (1979). Simple chemical reaction systems with limit cycle behaviour. J. Theor. Biol. 81, 389-400.

Thomas, D. (1975). Artificial enzyme membrane, transport, memory, and oscillatory phenomena, in Analysis and Control of Immobilised Enzyme Systems, D. Thomas and J. P. Kervenez (Eds), Berlin, Heidelberg, New York: Springer, pp. 115-150.

Thomas, R. D. K. (1976). Constraints of ligament growth, form and function on evolution in the Arcoida (Mollusca: Bivalvia). Paleobiology 2, 64-83.

Thomas, R. D. K., A. Madzvamuse, P. K. Maini and A. J. Wathen (2000). Growth Patterns of Noetiid Ligaments: Implications of Developmental Models for the Origin of an Evolutionary Novelty Among Arcoid Bivalves, The Evolutionary Biology of the Bivalvia, E. M. Harper, J. D. Taylor and J. A. Crame, (Eds) (Special Publications 177), Geological Society, London, pp. 289-279.

Thompson, D. W. (1917). On Growth and Form, 1st edn, Cambridge: Cambridge University Press.

Trueman, E. R. (1969). Ligament, in Treatise on Invertebrate Paleontology, R. C. Moore, (Ed.) Geological Society of America and University of Kansas (Part N, Mollusca 6), pp. 58-64.

Turing, A. M. (1952). The chemical basis of morphogenesis. Phil. Trans. Roy. Soc. Lond. B 237, 37-72. 